\title{
Towards learning universal, regional, and local hydrological behaviors via machine learning applied to large-sample datasets
}

\author{
Frederik Kratzert ${ }^{1}$, Daniel Klotz ${ }^{1}$, Guy Shalev ${ }^{2}$, Günter Klambauer ${ }^{1}$, Sepp Hochreiter , $^{1}$, and Grey Nearing ${ }^{3, *}$ \\ ${ }^{1}$ LIT AI Lab \& Institute for Machine Learning, Johannes Kepler University Linz, Linz, Austria \\ ${ }^{2}$ Google Research, Tel Aviv, Israel \\ ${ }^{3}$ Department of Geological Sciences, University of Alabama, Tuscaloosa, AL, USA \\ ${ }^{*}$ These authors contributed equally to this work.
}

Correspondence: Frederik Kratzert (kratzert@ml.jku.at)

Received: 15 July 2019 - Discussion started: 2 August 2019

Revised: 29 October 2019 - Accepted: 6 November 2019 - Published: 17 December 2019

\begin{abstract}
Regional rainfall-runoff modeling is an old but still mostly outstanding problem in the hydrological sciences. The problem currently is that traditional hydrological models degrade significantly in performance when calibrated for multiple basins together instead of for a single basin alone. In this paper, we propose a novel, data-driven approach using Long Short-Term Memory networks (LSTMs) and demonstrate that under a "big data" paradigm, this is not necessarily the case. By training a single LSTM model on 531 basins from the CAMELS dataset using meteorological time series data and static catchment attributes, we were able to significantly improve performance compared to a set of several different hydrological benchmark models. Our proposed approach not only significantly outperforms hydrological models that were calibrated regionally, but also achieves better performance than hydrological models that were calibrated for each basin individually. Furthermore, we propose an adaption to the standard LSTM architecture, which we call an Entity-Aware-LSTM (EA-LSTM), that allows for learning catchment similarities as a feature layer in a deep learning model. We show that these learned catchment similarities correspond well to what we would expect from prior hydrological understanding.
\end{abstract}

\section{Introduction}

A long-standing problem in the hydrological sciences is about how to use one model, or one set of models, to provide spatially continuous hydrological simulations across large areas (e.g., regional, continental, global). This is the socalled regional modeling problem, and the central challenge is about how to extrapolate hydrologic information from one area to another - e.g., from gauged to ungauged watersheds, from instrumented to non-instrumented hillslopes, or from areas with flux towers to areas without (Blöschl and Sivapalan, 1995). Often this is done using ancillary data (e.g., soil maps, remote sensing, digital elevation maps) to help understand similarities and differences between different areas. The regional modeling problem is thus closely related to the problem of prediction in ungauged basins (Blöschl et al., 2013; Sivapalan et al., 2003). This problem is well documented in several review papers; therefore, we point the interested reader to the comprehensive reviews by Razavi and Coulibaly (2013) and Hrachowitz et al. (2013) and to the more recent review in the introduction by Prieto et al. (2019).

Currently, the most successful hydrological models are calibrated to one specific basin, whereas a regional model must be somehow "aware" of differences between hydrologic behaviors in different catchments (e.g., ecology, geology, pedology, topography, geometry). The challenge of regional modeling is to learn and encode these differences so that differences in catchment characteristics translate into appropriately heterogeneous hydrologic behavior. 
Razavi and Coulibaly (2013) recognize two primary types of strategies for regional modeling: model-dependent methods and model-independent (data-driven) methods. Here, modeldependent denotes approaches where regionalization explicitly depends on a pre-defined hydrological model (e.g., classical process-based models), while model-independent denotes data-driven approaches that do not include a specific model. The critical difference is that the first tries to derive hydrologic parameters that can be used to run simulation models from available data (i.e., observable catchment characteristics). In this case, the central challenge is the fact that there is typically strong interaction between individual model parameters (e.g., between soil porosity and soil depth, or between saturated conductivity and an infiltration rate parameter), such that any meaningful joint probability distribution over model parameters will be complex and multimodal. This is closely related to the problem of equifinality (Beven and Freer, 2001).

Model-dependent regionalization has enjoyed major attention from the hydrological community, so that today a large variety of approaches exist. To give a few selective examples, Seibert (1999) calibrated a conceptual model for 11 catchments and regressed them against the available catchment characteristics. The regionalization capacity was tested against seven other catchments, where the reported performance ranged between an Nash-Sutcliffe efficiency (NSE) of 0.42 and 0.76. Samaniego et al. (2010) proposed a multiscale parameter regionalization (MPR) method, which simultaneously sets up the model and a regionalization scheme by regressing the global parameters of a set of a priori defined transfer functions that map from ancillary data like soil properties to hydrological model parameters. Beck et al. (2016) calibrated a conceptual model for 1787 catchments around the globe, used these as a catalog of "donor catchments", and then extended this library to new catchments by identifying the 10 most similar catchments from the library in terms of climatic and physiographic characteristics to parameterize a simulation ensemble. Prieto et al. (2019) first regionalized hydrologic signatures (Gupta et al., 2008) using a regression model (random forests) and then calibrated a rainfall-runoff model to the regionalized hydrologic signatures.

Model-independent methods, in contrast, do not rely on prior knowledge of the hydrological system. Instead, these methods learn the entire mapping from ancillary data and meteorological inputs to streamflow or other output fluxes directly. A model of this type has to "learn" how catchment attributes or other ancillary data distinguish between different catchment response behaviors. However, hydrological modeling typically provides the most accurate predictions when a model is calibrated to a single specific catchment (Mizukami et al., 2017), whereas data-driven approaches might benefit from a large cross section of diverse training data, because knowledge can be transferred across sites. Among the category of data-driven approaches are neural networks. Besaw et al. (2010) showed that an artificial neural network trained on one catchment (using only meteorological inputs) could be moved to a similar catchment (during a similar time period). However, the accuracy of their network in the training catchment was only a NSE of 0.29. Recently, Kratzert et al. (2018b) have shown that Long Short-Term Memory (LSTM) networks, a special type of recurrent neural network, are well suited for the task of rainfall-runoff modeling. This study already included the first experiments towards regional modeling while still using only meteorological inputs and ignoring ancillary catchment attributes. In a preliminary study Kratzert et al. (2018c) demonstrated that their LSTM-based approach outperforms, on average, the well-calibrated Sacramento Soil Moisture Accounting Model (SAC-SMA) in an asymmetrical comparison where the LSTM was used in an ungauged setting and SAC-SMA was used in a gauged setting - i.e., SAC-SMA was calibrated individually for each basin, whereas the LSTM never saw training data from any catchment where it was used for prediction. This was done by providing the LSTM-based model with meteorological forcing data and additional catchment attributes. From these preliminary results we can already assume that this general modeling approach is promising and has the potential for regionalization.

The objectives of this study are

i. to demonstrate that we can use large-sample hydrology data (Gupta et al., 2014; Peters-Lidard et al., 2017) to develop a regional rainfall-runoff model that capitalizes on observable ancillary data in the form of catchment attributes to produce accurate streamflow estimates over a large number of basins,

ii. to benchmark the performance of our neural network model against several existing hydrology models, and

iii. to show how the model uses information about catchment characteristics to differentiate between different rainfall-runoff behaviors.

To this end we built an LSTM-based model that learns catchment similarities directly from meteorological forcing data and ancillary data of multiple basins and evaluate its performance in a "gauged" setting, meaning that we never ask our model to predict in a basin where it did not see training data. Concretely, we propose an adaption of the LSTM where catchment attributes explicitly control which parts of the LSTM state space are used for a given basin. Because the model is trained using both catchment attributes and meteorological time series data, to predict streamflow, it can learn how to combine different parts of the network to simulate different types of rainfall-runoff behaviors. In principle, the approach explicitly allows for sharing of parts of the networks for similarly behaving basins while using different independent parts for basins with completely different rainfall-runoff behavior. Furthermore, our adaption provides a mapping from catchment attribute space into a 
learned, high-dimensional space, i.e., a so-called embedding, in which catchments with similar rainfall-runoff behavior can be placed together. This embedding can be used to preform data-driven catchment similarity analysis.

The paper is organized as follows. Section 2 (Methods) describes our LSTM-based model, the data, the benchmark hydrological models, and the experimental design. Section 3 (Results) presents our modeling results, the benchmarking results, and the results of our embedding layer analysis. Section 4 (Discussion and conclusion) reviews certain implications of our model and results and summarizes the advantages of using data-driven methods for extracting information from catchment observables for regional modeling.

\section{Methods}

\subsection{A brief overview of the Long Short-Term Memory network}

An LSTM network is a type of recurrent neural network that includes dedicated memory cells that store information over long time periods. A specific configuration of operations in this network, so-called gates, controls the information flow within the LSTM (Hochreiter, 1991; Hochreiter and Schmidhuber, 1997). These memory cells are, in a sense, analogous to a state vector in a traditional dynamical systems model, which makes LSTMs potentially an ideal candidate for modeling dynamical systems like watersheds. Compared to other types of recurrent neural networks, LSTMs do not have a problem with exploding and/or vanishing gradients, which allows them to learn long-term dependencies between input and output features. This is desirable for modeling catchment processes like snow accumulation and snowmelt that have relatively long timescales compared with the timescales of purely input-driven domains (i.e., precipitation events).

An LSTM works as follows (see also Fig. 1a): given an input sequence $\boldsymbol{x}=[\boldsymbol{x}[1], \ldots, \boldsymbol{x}[T]]$ with $T$ time steps, where each element $\boldsymbol{x}[t]$ is a vector containing input features (model inputs) at time step $t(1 \leq t \leq T)$, the following equations describe the forward pass through the LSTM:

$$
\begin{aligned}
\boldsymbol{i}[t] & =\sigma\left(\mathbf{W}_{\mathrm{i}} \boldsymbol{x}[t]+\mathbf{U}_{\mathrm{i}} \boldsymbol{h}[t-1]+\boldsymbol{b}_{\mathrm{i}}\right), \\
\boldsymbol{f}[t] & =\sigma\left(\mathbf{W}_{\mathrm{f}} \boldsymbol{x}[t]+\mathbf{U}_{\mathrm{f}} \boldsymbol{h}[t-1]+\boldsymbol{b}_{\mathrm{f}}\right), \\
\boldsymbol{g}[t] & =\tanh \left(\mathbf{W}_{\mathrm{g}} \boldsymbol{x}[t]+\mathbf{U}_{\mathrm{g}} \boldsymbol{h}[t-1]+\boldsymbol{b}_{\mathrm{g}}\right), \\
\boldsymbol{o}[t] & =\sigma\left(\mathbf{W}_{\mathrm{o}} \boldsymbol{x}[t]+\mathbf{U}_{\mathrm{o}} \boldsymbol{h}[t-1]+\boldsymbol{b}_{\mathrm{o}}\right), \\
\boldsymbol{c}[t] & =\boldsymbol{f}[t] \odot \boldsymbol{c}[t-1]+\boldsymbol{i}[t] \odot \boldsymbol{g}[t], \\
\boldsymbol{h}[t] & =\boldsymbol{o}[t] \odot \tanh (\boldsymbol{c}[t]),
\end{aligned}
$$

where $\boldsymbol{i}[t], \boldsymbol{f}[t]$, and $\boldsymbol{o}[t]$ are the input gate, forget gate, and output gate, respectively, $\boldsymbol{g}[t]$ is the cell input and $\boldsymbol{x}[t]$ is the network input at time step $t(1 \leq t \leq T)$, and $\boldsymbol{h}[t-1]$ is the recurrent input, $c[t-1]$ the cell state from the previous time step. At the first time step, the hidden and cell states are initialized as a vector of zeros. $\mathbf{W}, \mathbf{U}$, and $\boldsymbol{b}$ are learnable parameters for each gate, where subscripts indicate which gate the particular weight matrix/vector is used for, $\sigma(\cdot)$ is the sigmoid function, $\tanh (\cdot)$ is the hyperbolic tangent function, and $\odot$ is element-wise multiplication. The intuition behind this network is that the cell states $(\boldsymbol{c}[t])$ characterize the memory of the system. The cell states can get modified by the forget gate $(f[t])$, which can delete states, and the input gate $(i[t])$ and cell update $(g[t])$, which can add new information. In the latter case, the cell update is seen as the information that is added and the input gate controls into which cells new information is added. Finally, the output gate $(\boldsymbol{o}[t])$ controls which information, stored in the cell states, is outputted. For a more detailed description, as well as a hydrological interpretation, see Kratzert et al. (2018b).

\subsection{A new type of recurrent network: the Entity-Aware-LSTM}

To reiterate from the introduction, our objective is to build a network that learns to extract information that is relevant to rainfall-runoff behaviors from observable catchment attributes. To achieve this, it is necessary to provide the network with information on the catchment characteristics that contain some amount of information that allows for discrimination between different catchments. Ideally, we want the network to condition the processing of the dynamic inputs on a set of static catchment characteristics. That is, we want the network to learn a mapping from meteorological time series into streamflow that itself (i.e., the mapping) depends on a set of static catchment characteristics that could, in principle, be measured anywhere in our modeling domain.

One way to do this would be to add the static features as additional inputs at every time step. That is, we could simply augment the vectors $\boldsymbol{x}[t]$ at every time step with a set of catchment characteristics that do not (necessarily) change over time. However, this approach does not allow us to directly inspect what the LSTM learns from these static catchment attributes.

Our proposal is therefore to use a slight variation on the normal LSTM architecture (an illustration is given in Fig. 1b):

$$
\begin{aligned}
\boldsymbol{i} & =\sigma\left(\mathbf{W}_{\mathrm{i}} \boldsymbol{x}_{\mathrm{s}}+\boldsymbol{b}_{\mathrm{i}}\right), \\
\boldsymbol{f}[t] & =\sigma\left(\mathbf{W}_{\mathrm{f}} \boldsymbol{x}_{\mathrm{d}}[t]+\mathbf{U}_{\mathrm{f}} \boldsymbol{h}[t-1]+\boldsymbol{b}_{\mathrm{f}}\right), \\
\boldsymbol{g}[t] & =\tanh \left(\mathbf{W}_{\mathrm{g}} \boldsymbol{x}_{\mathrm{d}}[t]+\mathbf{U}_{\mathrm{g}} \boldsymbol{h}[t-1]+\boldsymbol{b}_{\mathrm{g}}\right), \\
\boldsymbol{o}[t] & =\sigma\left(\mathbf{W}_{\mathrm{o}} \boldsymbol{x}_{\mathrm{d}}[t]+\mathbf{U}_{\mathrm{o}} \boldsymbol{h}[t-1]+\boldsymbol{b}_{\mathrm{o}}\right), \\
\boldsymbol{c}[t] & =\boldsymbol{f}[t] \odot \boldsymbol{c}[t-1]+\boldsymbol{i} \odot \boldsymbol{g}[t], \\
\boldsymbol{h}[t] & =\boldsymbol{o}[t] \odot \tanh (\boldsymbol{c}[t]) .
\end{aligned}
$$

Here $\boldsymbol{i}$ is an input gate, which now does not change over time. $\boldsymbol{x}_{\mathrm{s}}$ are the static inputs (e.g., catchment attributes) and $\boldsymbol{x}_{\mathrm{d}}[t]$ are the dynamic inputs (e.g., meteorological forcings) at time step $t(1 \leq t \leq T)$. The rest of the LSTM remains unchanged. The intuition is as follows: we explicitly process the static 
(a) LSTM

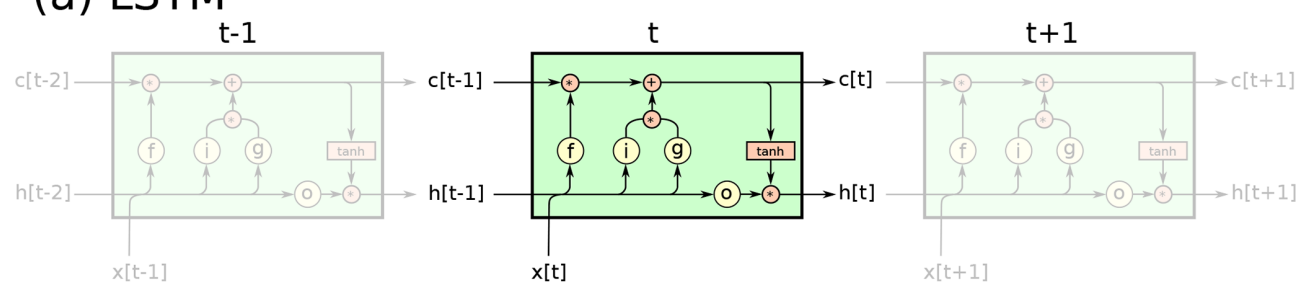

(b) EA-LSTM

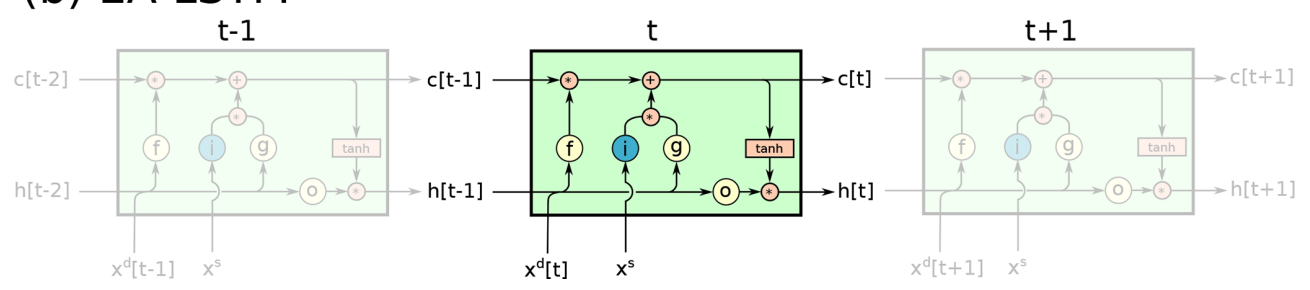

Figure 1. Visualization of (a) the standard (LSTM) cell as defined by Eqs. (1)-(6) and (b) the proposed Entity-Aware-LSTM (EA-LSTM) cell as defined by Eqs. (7)-(12).

inputs $\boldsymbol{x}_{\mathrm{s}}$ and the dynamic inputs $\boldsymbol{x}_{\mathrm{d}}[t]$ separately within the architecture and assign them special tasks. The static features control, through input gate $(i)$, which parts of the LSTM are activated for any individual catchment, while the dynamic and recurrent inputs control what information is written into the memory $(g[t])$, what is deleted $(f[t])$, and what of the stored information to output $(\boldsymbol{o}[t])$ at the current time step $t$.

We call this an Entity-Aware-LSTM (EA-LSTM) because it explicitly differentiates between similar types of dynamical behaviors (here rainfall-runoff processes) that differ between individual entities (here different watersheds). After training, the static input gate of the EA-LSTM contains a series of real values in the range $(0,1)$ that allow certain parts of the input gate to be active through the simulation of any individual catchment. In principle, different groups of catchments can share different parts of the full trained network.

This is an embedding layer, which allows for non-naive information sharing between the catchments. For example, we could potentially discover, after training, that two particular catchments share certain parts of the activated network based on geological similarities while other parts of the network remain distinct due to ecological dissimilarities. This embedding layer allows for complex interactions between catchment characteristics, and - importantly - makes it possible for those interactions to be directly informed by the rainfallrunoff data from all catchments used for training.

\subsection{Objective function: a smooth-joint NSE}

An objective function is required for training the network. For regression tasks such as runoff prediction, the meansquared error (MSE) is commonly used. Hydrologists also sometimes use the NSE because it has an interpretable range of $(-\infty, 1)$. Both the MSE and NSE are squared error loss functions, with the difference being that the latter is normal- ized by the total variance of the observations. For singlebasin optimization, the MSE and NSE will typically yield the same optimum parameter values, discounting any effects in the numerical optimizer that depend on the absolute magnitude of the loss value.

The linear relation between these two metrics (MSE and NSE) is lost, however, when calculated over data from multiple basins. In this case, the means and variances of the observation data are no longer constant because they differ between basins. We will exploit this fact. In our case, the MSE from a basin with low average discharge (e.g., smaller, arid basins) is generally smaller than the MSE from a basin with high average discharge (e.g., larger, humid basins). We need an objective function that does not depend on basin-specific mean discharge so that we do not overweight large humid basins (and thus perform poorly on small, arid basins). Our loss function is therefore the average of the NSE values calculated at each basin that supplies training data - referred to as basin-averaged Nash-Sutcliffe efficiency (NSE*). Additionally, we add a constant term to the denominator $(\epsilon=0.1)$, the variance of the observations, so that our loss function does not explode (to negative infinity) for catchments with very low flow variance. Our loss function is therefore

$\mathrm{NSE}^{*}=\frac{1}{B} \sum_{b=1}^{B} \sum_{n=1}^{N} \frac{\left(\widehat{y}_{n}-y_{n}\right)^{2}}{(s(b)+\epsilon)^{2}}$,

where $B$ is the number of basins, $N$ is the number of samples (days) per basin $B, \widehat{y}_{n}$ is the prediction of sample $n$ $(1 \leq n \leq N), y_{n}$ is the observation, and $s(b)$ is the standard deviation of the discharge in basin $b(1 \leq b \leq B)$, calculated from the training period. In general, an entity-aware deep learning model will need a loss function that does not underweight entities with lower (relative to other entities in the training dataset) absolute values in the target data. 
(a) Mean elevation

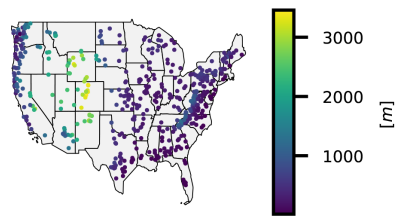

(c) Forest fraction

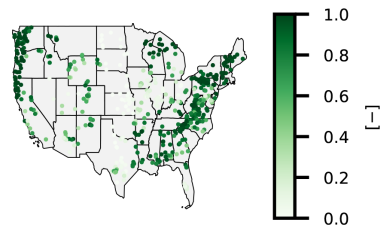

(b) Aridity

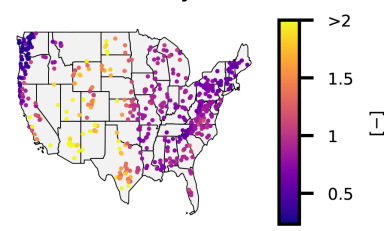

(d) Mean daily precipitation

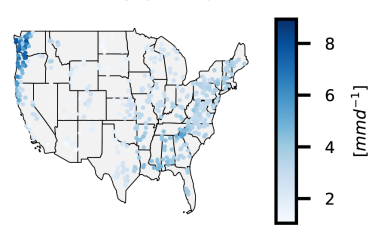

Figure 2. Overview of the basin location and corresponding catchment attributes. (a) The mean catchment elevation, (b) the catchment aridity (PET/P), (c) the fraction of the catchment covered by forest, and (d) the daily average precipitation.

\subsection{The NCAR CAMELS dataset}

To benchmark our proposed EA-LSTM model and to assess its ability to learn meaningful catchment similarities, we will use the Catchment Attributes and Meteorological (CAMELS) dataset (Newman et al., 2014; Addor et al., 2017b). CAMELS is a set of data concerning 671 basins that is curated by the US National Center for Atmospheric Research (NCAR). The CAMELS basins range in size between 4 and $25000 \mathrm{~km}^{2}$ and were chosen because they have relatively low anthropogenic impacts. These catchments span a range of geologies and ecoclimatologies, as described in Newman et al. (2015) and Addor et al. (2017a).

We used the same subselection of 531 basins from the CAMELS dataset that was used by Newman et al. (2017). These basins are mapped in Fig. 2 and were chosen (by Newman et al., 2017) out of the full set because some of the basins have a large (>10\%) discrepancy between different strategies for calculating the basin area, and incorrect basin area would introduce significant uncertainty into a modeling study. Furthermore, only basins with a catchment area smaller than $2000 \mathrm{~km}^{2}$ were kept.

For time-dependent meteorological inputs $\left(\boldsymbol{x}_{\mathrm{d}}[t]\right)$, we used the daily, basin-averaged Maurer forcings (Wood et al., 2002) supplied with CAMELS. Our input data include (i) daily cumulative precipitation, (ii) daily minimum air temperature, (iii) daily maximum air temperature, (iv) average short-wave radiation, and (v) vapor pressure. Furthermore, 27 CAMELS catchment characteristics were used as static input features $\left(\boldsymbol{x}_{\mathrm{s}}\right)$; these were chosen as a subset of the full set of characteristics explored by Addor et al. (2017b) that are derivable from remote sensing or CONUS-wide available data products. These catchment attributes include climatic and vegetation indices, as well as soil and topographical properties (see Table A1 for an exhaustive list).

\subsection{Benchmark models}

The first part of this study benchmarks our proposed model against several high-quality benchmarks. The purpose of this exercise is to show that the EA-LSTM provides reasonable hydrological simulations.

To do this, we collected a set of existing hydrological models ${ }^{1}$ that were configured, calibrated, and run by several previous studies over the CAMELS catchments. These models are (i) SAC-SMA (Burnash et al., 1973; Burnash, 1995) coupled with the Snow-17 snow routine (Anderson, 1973), hereafter referred to as SAC-SMA, (ii) VIC (Liang et al., 1994), (iii) FUSE (Clark et al., 2008; Henn et al., 2008) (three different model structures, 900, 902, 904), (iv) HBV (Seibert and Vis, 2012), and (v) mHM (Samaniego et al., 2010; Kumar et al., 2013). In some cases, these models were calibrated to individual basins, and in other cases they were not. All of these benchmark models were run by other groups - we did not run any of our own benchmarks. We chose to use existing model runs so as not to bias the calibration of the benchmarks to possibly favor our own model. Each set of simulations that we used for benchmarking is documented elsewhere in the hydrology literature (references below). Each of these benchmark models use the same daily Maurer forcings that we used with our EA-LSTM, and all were calibrated and validated on the same time period(s). These benchmark models can be distinguished into two different groups.

1. Models calibrated for each basin individually. These are SAC-SMA (Newman et al., 2017), VIC (Newman et al., 2017), $\mathrm{FUSE}^{2}$, mHM (Mizukami et al., 2019), and HBV (Seibert et al., 2018). The HBV model supplied both a lower and an upper benchmark, where the lower benchmark is an ensemble mean of 1000 uncalibrated HBV models and the upper benchmark is an ensemble of 100 calibrated HBV models.

2. Models that were regionally calibrated. These share one parameter set for all basins in the dataset. Here we have calibrations of the VIC model (Mizukami et al., 2017) and mHM (Rakovec et al., 2019).

\subsection{Experimental setup}

All model calibration and training were performed using data from the time period 1 October 1999 through 30 September 2008. All model and benchmark evaluation was done using data from the time period 1 October 1989 through 30 September 1999. We trained a single LSTM or EA-LSTM

\footnotetext{
${ }^{1}$ Will be released on HydroShare (https://doi.org/10.4211/hs.474ecc37e7db45baa425cdb4fc1b61e1).

${ }^{2}$ The FUSE runs were generated by Nans Addor (n.addor@uea.ac.uk) and given to us by personal communication. These runs are part of current development by N. Addor on the FUSE model itself and might not reflect the final performance of the FUSE model.
} 
model using calibration period data from all basins and evaluated this model using validation period data from all basins. This implies that a single parameter set (i.e., $\mathbf{W}, \mathbf{U}, \boldsymbol{b}$ from Eqs. 1-4 and 7-10) was trained to work across all basins.

We trained and tested the following three model configurations.

- LSTM without static inputs. A single LSTM trained on the combined calibration data from all basins, using only the meteorological forcing data and ignoring static catchment attributes.

- LSTM with static inputs. A single LSTM trained on the combined calibration data of all basins, using the meteorological features as well as the static catchment attributes. These catchment descriptors were concatenated to the meteorological inputs at each time step.

- EA-LSTM with static inputs. A single EA-LSTM trained on the combined calibration data of all basins, using the meteorological features as well as the static catchment attributes. The catchment attributes were input to the static input gate in Eq. (7), while the meteorological inputs were used at all remaining parts of the network (Eqs. 8-10).

All three model configurations were trained using the squared-error performance metrics discussed in Sect. 2.3 (MSE and NSE*). This resulted in six different model/training configurations.

To account for stochasticity in the network initialization and in the optimization procedure (we used stochastic gradient descent), all networks were trained with $n=8$ different random seeds. Predictions from the different seeds were combined into an ensemble by taking the mean prediction at each time step of all $n$ different models under each configuration. In total, we trained and tested six different settings and eight different models per setting for a total of 48 different trained LSTM-type models. For all LSTMs we used the same architecture (apart from the inclusion of a static input gate in the EA-LSTM), which we found through hyperparameter optimization (see Appendix B for more details about the hyperparameter search). The LSTMs had 256 memory cells and a single fully connected layer with a dropout rate (Srivastava et al., 2014) of 0.4. The LSTMs were run in sequence-tovalue mode (as opposed to sequence-to-sequence mode), so that to predict a single (daily) discharge value required meteorological forcings from 269 preceding days, as well as the forcing data of the target day, making the input sequences 270 time steps long.

\subsubsection{Assessing model performance}

Because no one evaluation metric can fully capture the consistency, reliability, accuracy, and precision of a streamflow model, it was necessary to use a variety of performance metrics for model benchmarking (Gupta et al., 1998). Evalua- tion metrics used to compare models are listed in Table 1. These metrics focus specifically on assessing the ability of the model to capture high flows and low flows as well as on assessing overall performance using a decomposition of the standard squared error metrics that is less sensitive to bias (Gupta et al., 2009).

\subsubsection{Robustness and feature ranking}

All catchment attributes used in this study are derived from gridded data products (Addor et al., 2017a). Taking the catchment's mean elevation as an example, we would get different mean elevations depending on the resolution of the gridded digital elevation model. More generally, there is uncertainty in all CAMELS catchment attributes. Thus, it is important that we evaluate the robustness of our model and of our embedding layer (particular values of the 256 static input gates) to changes in the exact values of the catchment attributes. Additionally, we want some idea about the relative importance of different catchment attributes.

To estimate the robustness of the trained model to uncertainty in the catchment attributes, we added Gaussian noise $\mathcal{N}(0, \sigma)$ with increasing standard deviation to the individual attribute values and assessed resulting changes in model performance for each noise level. Concretely, additive noise was drawn from normal distributions with 10 different standard deviations: $\sigma=[0.1,0.2, \ldots, 0.9,1.0]$. All input features (both static and dynamic) were standardized (zero mean, unit variance) before training, so these perturbation sigmas did not depend on the units or relative magnitudes of the individual catchment attributes. For each basin and each standard deviation we drew 50 random noise vectors, resulting in $531 \times 10 \times 50=265500$ evaluations of each trained EA-LSTM.

To provide a simple estimate of the most important static features of the trained model, we used the method of Morris (1991). Although the Morris method is relatively simple, it has been shown to provide meaningful estimations of the global sensitivity and is widely used (e.g., Herman et al., 2013; Wang and Solomatine, 2019). The method of Morris uses an approximation of local derivatives, which can be extracted directly from neural networks without additional computations, which makes this a highly efficient method of sensitivity analysis.

The method of Morris typically estimates feature sensitivities $\left(\mathrm{EE}_{i}\right)$ from local (numerical) derivatives.

$\mathrm{EE}_{i}=\frac{f\left(x_{1}, \ldots, x_{i}+\triangle_{i}, \ldots, x_{p}\right)-f(x)}{\triangle_{i}}$

Neural networks are completely differentiable (to allow for back-propagation) and thus it is possible to calculate the exact gradient with respect to the static input features. Thus, for neural networks the method of Morris can be applied analyt- 
Table 1. Overview of used evaluation metrics. The notation of the original publications is kept.

\begin{tabular}{|c|c|c|}
\hline Metric & Reference & Equation \\
\hline Nash-Sutcliffe efficiency (NSE) & Nash and Sutcliffe (1970) & $1-\frac{\sum_{t=1}^{T}\left(Q_{\mathrm{m}}[t]-Q_{\mathrm{o}}[t]\right)^{2}}{\sum_{t=1}^{T}\left(Q_{\mathrm{o}}[t]-\overline{Q_{\mathrm{o}}}\right)^{2}}$ \\
\hline$\alpha$-NSE decomposition & Gupta et al. (2009) & $\sigma_{\mathrm{s}} / \sigma_{\mathrm{o}}$ \\
\hline$\beta$-NSE decomposition & Gupta et al. (2009) & $\left(\mu_{\mathrm{s}}-\mu_{\mathrm{o}}\right) / \sigma_{\mathrm{o}}$ \\
\hline Top $2 \%$ peak flow bias (FHV) & Yilmaz et al. (2008) & $\frac{\sum_{h=1}^{H}\left(Q S_{h}-Q O_{h}\right)}{\sum_{h=1}^{H} Q O_{h}} \times 100$ \\
\hline Bias of FDC midsegment slope (FMS) & Yilmaz et al. (2008) & $\frac{\left(\log \left(Q S_{\mathrm{m} 1}\right)-\log \left(Q S_{\mathrm{m} 2}\right)\right)-\left(\log \left(Q O_{\mathrm{m} 1}\right)-\log \left(Q O_{\mathrm{m} 2}\right)\right)}{\left(\log \left(Q O_{\mathrm{m} 1}\right)-\log \left(Q O_{\mathrm{m} 2}\right)\right)} \times 100$ \\
\hline $30 \%$ low flow bias (FLV) & Yilmaz et al. (2008) & $\frac{\sum_{l=1}^{L}\left(\log \left(Q S_{l}\right)-\log \left(Q S_{L}\right)\right)-\sum_{l=1}^{L}\left(\log \left(Q O_{l}\right)-\log \left(Q O_{L}\right)\right)}{\sum_{l=1}^{L}\left(\log \left(Q O_{l}\right)-\log \left(Q O_{L}\right)\right)} \times 100$ \\
\hline
\end{tabular}

ically.

$\mathrm{EE}_{i}=\lim _{\triangle_{i} \rightarrow 0} \frac{f\left(x_{1}, \ldots, x_{i}+\triangle_{i}, \ldots, x_{p}\right)-f(x)}{\triangle_{i}}=\frac{\partial f(x)}{\partial x_{i}}$

This makes it unnecessary to run computationally expensive sampling methods to approximate the local gradient. Further, since we predict one time step of discharge at the time, we obtain this sensitivity measure for each static input for each day in the validation period. A global sensitivity measure for each basin and each feature is then derived by taking the average absolute gradient (Saltelli et al., 2004).

\subsubsection{Analysis of catchment similarity from the embedding layer}

Once the model is trained, the input gate vector ( $i$; see Eq. 7) for each catchment is fixed for the simulation period. This results in a vector that represents an embedding of the static catchment features (here in $\mathbb{R}_{27}$ ) into the high-dimensional space of the LSTM (here in $\mathbb{R}_{256}$ ). The result is a set of realvalued numbers that map the catchment characteristics onto a strength, or weight, associated with each particular cell state in the EA-LSTM. This weight controls how much of the cell input ( $g[t]$; see Eq. 9) is written into the corresponding cell state $(c[t]$; see Eq. 11).

Per design, our hypothesis is that the EA-LSTM will learn to group similar basins together into the high-dimensional space, so that hydrologically similar basins use similar parts of the LSTM cell states. This is dependent, of course, on the information content of the catchment attributes used as inputs, but the model should at least not degrade the quality of this information and should learn hydrologic similarity in a way that is useful for rainfall-runoff prediction. We tested this hypothesis by analyzing the learned catchment embedding from a hydrological perspective. We analyzed geographical similarity by using $k$-means clustering on the $\mathbb{R}_{256}$ feature space of the input gate embedding to delineate basin groupings and then plotted the clustering results geographically. The number of clusters was determined using a mean silhouette score.

In addition to visually analyzing the $k$-means clustering results by plotting them spatially (to ensure that the input embedding preserved expected geographical similarity), we measured the ability of these cluster groupings to explain variance in certain hydrological signatures in the CAMELS basins. For this, we used 13 of the hydrologic signatures that were used by Addor et al. (2018): (i) mean annual discharge ( $q$ mean), (ii) runoff ratio, (iii) slope of the flow duration curve (slope-fdc), (iv) baseflow index, (v) streamflow-precipitation elasticity (stream-elas), (vi) 5th percentile flow $\left(q_{5}\right)$, (vii) 95th percentile flow $\left(q_{95}\right)$, (viii) frequency of high-flow days (high-q-freq), (ix) mean duration of high-flow events (high-q-dur), (x) frequency of low-flow days (low-q-freq), (xi) mean duration of low-flow events (low-q-dur), (xii) zero flow frequency (zero-q-freq), and (xiii) average day of year when half of the cumulative annual flow occurs (mean-hfd).

Finally, we reduced the dimension of the input gate embedding layer (from $\mathbb{R}_{256}$ to $\mathbb{R}_{2}$ ) so as to be able to visualize dominant features in the input embedding. To do this we use a dimension reduction algorithm, called UMAP (McInnes et al., 2018) for "Uniform Manifold Approximation and Projection for Dimension Reduction”. UMAP is based on neighbor graphs (while, e.g., principle component analysis is based on matrix factorization), and it uses ideas from topological data analysis and manifold learning techniques to guarantee that information from the high-dimensional space is preserved in the reduced space. For further details we refer the reader to the original publication by McInnes et al. (2018). 


\section{Results}

This section is organized as follows.

- The first subsection (Sect. 3.1) presents a comparison between the three different LSTM-type model configurations discussed in Sect. 2.6.1. The emphasis in this comparison is to examine the effect of adding catchment attributes as additional inputs to the LSTM using the standard vs. adapted EA-LSTM architectures.

- The second subsection (Sect. 3.2) presents results from our benchmarking analysis, that is, the direct comparison between the performances of our EA-LSTM model with the full set of benchmark models outlined in Sect. 2.5.

- The third subsection (Sect. 3.3) presents results of the sensitivity analysis outlined in Sect. 2.6.2.

- The final subsection (Sect. 3.4) presents an analysis of the EA-LSTM embedding layer to demonstrate that the model learned how to differentiate between different rainfall-runoff behaviors across different catchments.

\subsection{Comparison between LSTM modeling approaches}

The key results from a comparison between the LSTM approaches are in Fig. 3, which shows the cumulative density functions (CDFs) of the basin-specific NSE values for all six LSTM models (three model configurations and two loss functions) over the 531 basins.

Table 2 contains average key overall performance statistics. Statistical significance was evaluated using the paired Wilcoxon test (Wilcoxon, 1945), and the effect size was evaluated using Cohen's $d$ (Cohen, 1988). The comparison contains four key results.

i. Using catchment attributes as static input features improves overall model performance as compared with not providing the model with catchment attributes. This is expected, but worth confirming.

ii. Training against the basin-average $\mathrm{NSE}^{*}$ loss function improves overall model performance as compared with training against an MSE loss function, especially in the low NSE spectra.

iii. There is statistically significant difference between the performance of the standard LSTM with static input features and the EA-LSTM, however, with a small effect size.

iv. Some of the error in the LSTM-type models is due to randomness in the training procedure and can be mitigated by running model ensembles.

Related to result (i), there was a significant difference between LSTMs with standard architecture trained with vs.

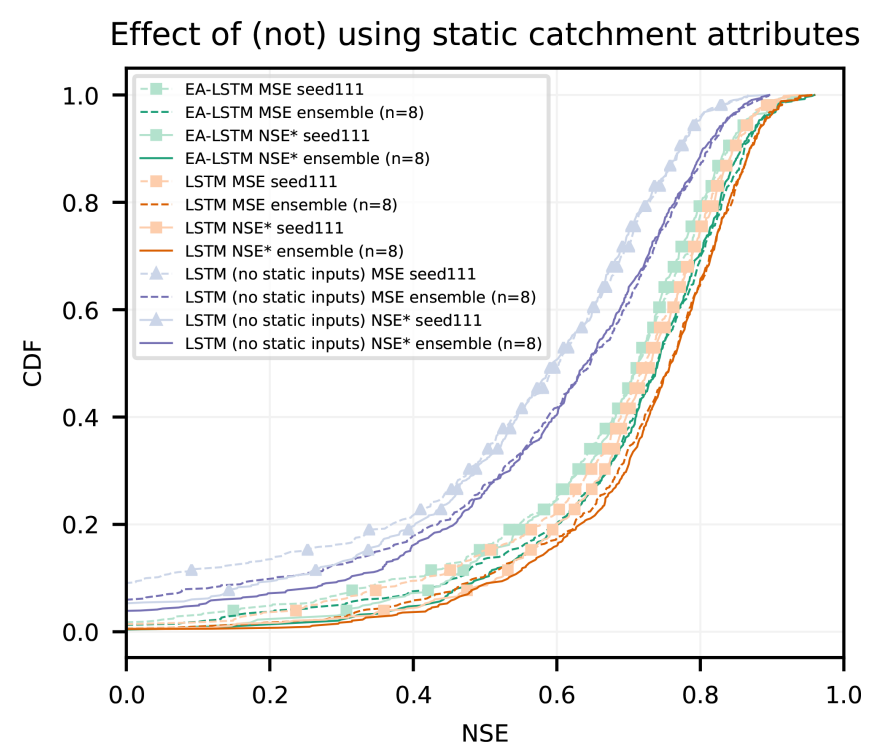

Figure 3. Cumulative density functions of the NSE for all LSTMtype model configurations described in Sect. 2.6.1. For each model type the ensemble mean and one of the $n=8$ repetitions are shown. LSTM configurations are shown in orange (with catchment attributes) and purple (without catchment attributes), and the EALSTM configurations (always with catchment attributes) are shown in green.

without static features (square vs. triangle markers in Fig. 3). The mean (over basins) NSE improved in comparison with the LSTM that did not take catchment characteristics as inputs by 0.44 (range $(0.38,0.56)$ ) when optimized using the MSE and 0.30 (range $(0.22,0.43)$ ) when optimized using the basin-average NSE* . To assess statistical significance for single models, we first calculated the mean basin performance, i.e., the mean SE per basin across the eight repetitions. The mean basin performance thus derived was then used for the test of significance. To assess statistical significance for ensemble means, the ensemble prediction (i.e., the mean discharge prediction of the eight model repetitions) was used to compare between different model approaches. For models trained using the standard MSE loss function, the $p$ value for the single model was $p=1.2 \times 10^{-75}$ and the $p$ value between the ensemble means was $p=4 \times 10^{-68}$. When optimized using the basin-average $\mathrm{NSE}^{*}$, the $p$ value for the single model was $p=8.8 \times 10^{-81}$ ) and the $p$ value between the ensemble means was $p=3.3 \times 10^{-75}$.

It is worth emphasizing that the improvement in overall model performance due to including catchment attributes implies that these attributes contain information that helps to distinguish different catchment-specific rainfall-runoff behaviors. This is especially interesting since these attributes are derived from remote sensing and other ubiquitously available data products, as described by Addor et al. (2017b). Our benchmarking analysis presented in the next subsection (Sect. 3.2) shows that this information content is sufficient 
Table 2. Evaluation results of the single models and ensemble means.

\begin{tabular}{|c|c|c|c|}
\hline \multirow[t]{2}{*}{ Model } & \multicolumn{2}{|c|}{$\mathrm{NSE}^{*}$} & \multirow[t]{2}{*}{ No. of basins with NSE $\leq 0$} \\
\hline & mean & median & \\
\hline \multicolumn{4}{|l|}{ LSTM without static inputs } \\
\hline \multicolumn{4}{|l|}{ using MSE: } \\
\hline Single model: & $0.24( \pm 0.049)$ & $0.60( \pm 0.005)$ & $44( \pm 4)$ \\
\hline $\begin{array}{l}\text { Ensemble mean }(n=8) \text { : } \\
\text { using NSE*: }\end{array}$ & 0.36 & 0.65 & 31 \\
\hline Single model: & $0.39( \pm 0.059)$ & $0.59( \pm 0.008)$ & $28( \pm 3)$ \\
\hline Ensemble mean $(n=8)$ : & 0.49 & 0.64 & 20 \\
\hline \multicolumn{4}{|l|}{ LSTM with static inputs } \\
\hline \multicolumn{4}{|l|}{ using MSE: } \\
\hline Single model: & $0.66( \pm 0.012)$ & $0.73( \pm 0.003)$ & $6( \pm 2)$ \\
\hline $\begin{array}{l}\text { Ensemble mean }(n=8) \text { : } \\
\text { using NSE*: }\end{array}$ & 0.71 & 0.76 & 3 \\
\hline Single model: & $0.69( \pm 0.013)$ & $0.73( \pm 0.002)$ & $2( \pm 1)$ \\
\hline Ensemble mean $(n=8)$ : & 0.72 & 0.76 & 2 \\
\hline \multicolumn{4}{|l|}{$E A-L S T M$} \\
\hline \multicolumn{4}{|l|}{ using MSE: } \\
\hline Single model: & $0.63( \pm 0.018)$ & $0.71( \pm 0.005)$ & $9( \pm 1)$ \\
\hline $\begin{array}{l}\text { Ensemble mean }(n=8) \text { : } \\
\text { using NSE*: }\end{array}$ & 0.68 & 0.74 & 6 \\
\hline Single model: & $0.67( \pm 0.006)$ & $0.71( \pm 0.005)$ & $3( \pm 1)$ \\
\hline Ensemble mean $(n=8)$ : & 0.70 & 0.74 & 2 \\
\hline
\end{tabular}

to perform high-quality regional modeling (i.e., competitive with lumped models calibrated separately for each basin).

Related to result (ii), using the basin-average NSE$^{*}$ loss function instead of a standard MSE loss function improved performance for single models (different individual seeds) as well as for the ensemble means across all model configurations (see Table 2). The differences are most pronounced for the EA-LSTM and for the LSTM without static features. For the EA-LSTM, the mean NSE for the single model increased from 0.63 when optimized with MSE to 0.67 when optimized with the basin average NSE*. For the LSTM trained without catchment characteristics the mean NSE went from 0.23 when optimized with MSE to 0.39 when optimized with $\mathrm{NSE}^{*}$. Further, the median NSE did not change significantly depending on loss function due to the fact that the improvements from using the $\mathrm{NSE}^{*}$ are mostly to performance in basins at the lower end of the NSE spectra (see also Fig. 1 dashed vs. solid lines). This is as expected as catchments with relatively low average flows have a small influence on (LSTM) training with an MSE loss function, which results in poor performance in these basins. Using the NSE* loss function helps to mitigate this problem. It is important to note that this is not the only reason why certain catchments have low skill scores, which can happen for a variety of reasons with any type of hydrological model (e.g., bad input data, unique catchment behaviors). This improvement at the lowperformance end of the spectrum can also been seen by looking at the number of "catastrophic failures", i.e., basins with an NSE value of less than zero. Across all models we see a reduction in this number when optimized with the basin average $\mathrm{NSE}^{*}$, compared to optimizing with MSE.

Related to result (iii), Fig. 3 shows a small difference in the empirical CDFs between the standard LSTM with static input features and the EA-LSTM under both functions (compare green vs. orange lines). The difference is significant ( $p$ value for single model $p=1 \times 10^{-28}, p$ value for the ensemble mean $p=2.1 \times 10^{-26}$, paired Wilcoxon test); however, the effect size is small: $d=0.055$. This is important because the embedding layer in the EA-LSTM adds a layer of interpretability to the LSTM, which we argue is desirable for scientific modeling in general and is useful in our case for understanding catchment similarity. This is only useful, however, if the EA-LSTM does not sacrifice performance compared to the less interpretable traditional LSTM. There is some small performance sacrifice in this case, likely due to an increase in the number of tunable parameters in the network, but the benefit of this small reduction in performance is explicability. 
Related to result (iv), in all cases there were several basins with very low NSE values (this is also true for the benchmark models, which we will discuss in Sect. 3.2). Using catchment characteristics as static input features with the EA-LSTM architecture reduced the number of such basins from 44 (31) to 9 (6) for the average single model (ensemble mean) when optimized with the MSE and from 28 (20) to 3 (2) for the average single model (ensemble mean) if optimized using the basin-average NSE* ${ }^{*}$ This result is worth emphasizing: each LSTM or EA-LSTM trained over all basins results in a certain number of basins that perform poorly (NSE $\leq 0)$, but the basins where this happens are not always the same. The model outputs, and therefore the number of catastrophic failures, differ depending on the randomness in the weight initialization and optimization procedure and, thus, running an ensemble of LSTMs substantively reduces this effect. This is good news for deep learning - it means that at least a portion of uncertainty can be mitigated using model ensembles. We leave as an open question for future research how many ensemble members, as well as how these are initialized, should be used to minimize uncertainty for a given dataset.

\subsection{Model benchmarking: EA-LSTM vs. calibrated hydrology models}

The results in this section are calculated from 447 basins that were modeled by all benchmark models, as well as our EALSTM. In this section, we concentrate on benchmarking the EA-LSTM; however, for the sake of completeness, we added the results of the LSTM with static inputs to all figures and tables.

First we compared the EA-LSTM against the two hydrological models that were regionally calibrated (VIC and mHM). Specifically, what was calibrated for each model was a single set of transfer functions that map from static catchment characteristics to model parameters. The procedure for parameterizing these models for regional simulations is described in detail by the original authors: Mizukami et al. (2017) for VIC and Rakovec et al. (2019) for mHM. Figure 4 shows that the EA-LSTM outperformed both regionally calibrated benchmark models by a large margin. Even the LSTM trained without static catchment attributes (only trained on meteorological forcing data) outperformed both regionally calibrated models consistently as a single model, and even more so as an ensemble.

The mean and median NSE scores across the basins of the individual EA-LSTM models $\left(N_{\text {ensemble }}=8\right)$ were $0.67 \pm$ $0.006(0.71)$ and $0.71 \pm 0.004(0.74)$, respectively. In contrast, VIC had a mean NSE of 0.17 and a median NSE of 0.31 and the mHM had a mean NSE of 0.44 and a median NSE of 0.53. Overall, VIC scored higher than the EA-LSTM ensemble in 2 out of 447 basins $(0.4 \%)$ and mHM scored higher than the EA-LSTM ensemble in 16 basins (3.58\%). Investigating the number of catastrophic failures (the number of basins where NSE $\leq 0$ ), the average single EA-LSTM failed

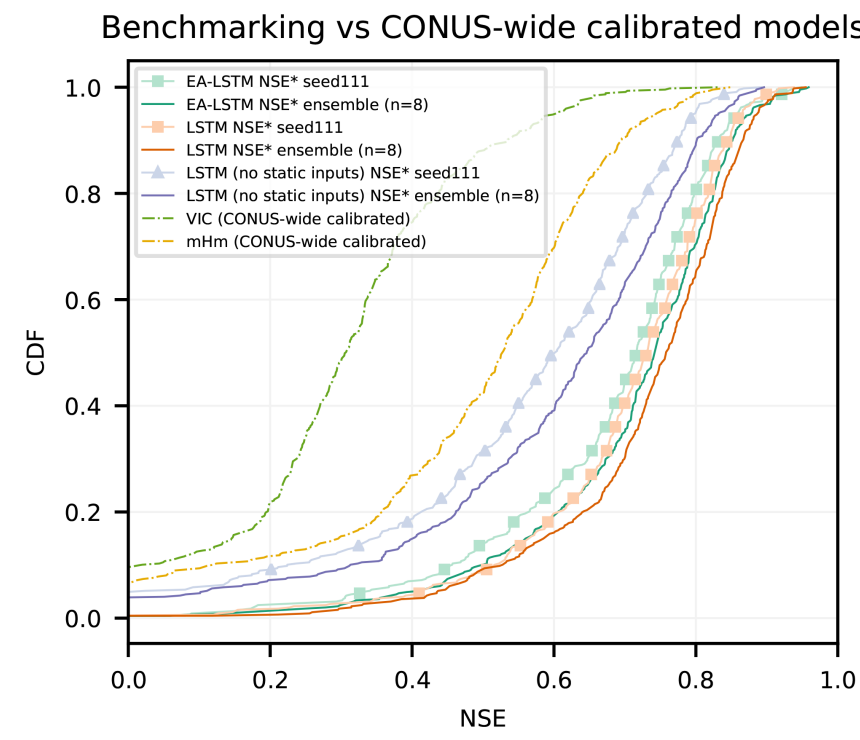

Figure 4. Cumulative density functions of the NSE of two regionally calibrated benchmark models (VIC and mHM), compared to the EA-LSTM and the LSTM trained with and without static input features.

in approximately 2 basins out of 447 basins $(0.4 \pm 0.2 \%)$ and the ensemble mean of the EA-LSTM failed in only a single basin (i.e., $0.2 \%$ ). In comparison, mHM failed in 29 basins $(6.49 \%)$ and VIC failed in 41 basins $(9.17 \%)$.

Second, we compared our multi-basin calibrated EALSTMs to individual-basin calibrated hydrological models. This is a more rigorous benchmark than the regionally calibrated models, since hydrological models usually perform better when trained for specific basins. Figure 5 compares CDFs of the basin-specific NSE values for all benchmark models over the 447 basins. Table 3 contains the performance statistics for these benchmark models as well as for the recalculated EA-LSTM.

The main benchmarking result is that the EA-LSTM significantly outperforms all benchmark models in the overall NSE. The two best-performing hydrological models were the ensemble $(n=100)$ of basin-calibrated HBV models and a single basin-calibrated mHM model. The EA-LSTM outperformed both of these models at any reasonable alpha level. The $p$ value for the single model, compared to the HBV upper bound, was $p=1.9 \times 10^{-4}$ and for the ensemble mean $p=6.2 \times 10^{-11}$ with a medium effect size (Cohen's $d$ for single model $d=0.22$ and for the ensemble mean $d=0.40$ ). The $p$ value for the single model, compared to the basinwise calibrated mHM, was $p=4.3 \times 10^{-6}$ and for the ensemble mean $p=1.0 \times 10^{-13}$ with a medium effect size (Cohen's $d$ for single model $d=0.26$ and for the ensemble mean $d=0.45$ ).

Regarding all other metrics except the Kling-Gupta decomposition of the NSE, there was no statistically significant difference between the EA-LSTM and the two best- 
Table 3. Comparison of the EA-LSTM and LSTM (with static inputs) average single model and ensemble mean to the full set of benchmark models. VIC (basin) and mHM (basin) denote the basin-wise calibrated models, while VIC (CONUS) and mHM (CONUS) denote the CONUS-wide calibrated models. HBV (lower) denotes the ensemble mean of $n=1000$ uncalibrated HBVs, while HBV (upper) denotes the ensemble mean of $n=100$ calibrated HBVs (for details, see Seibert et al., 2018). For the FUSE model, the numbers behind the name denote different FUSE model structures. All statistics were calculated from the validation period of all 447 commonly modeled basins.

\begin{tabular}{|c|c|c|c|c|c|c|c|c|}
\hline \multirow[t]{2}{*}{ Model } & \multicolumn{2}{|c|}{$\mathrm{NSE}^{\mathrm{a}}$} & \multirow{2}{*}{$\begin{array}{l}\text { No. of basins } \\
\text { with NSE } \leq 0\end{array}$} & \multirow{2}{*}{$\begin{array}{c}\alpha-\mathrm{NSE}^{\mathrm{b}} \\
\text { median }\end{array}$} & \multirow{2}{*}{$\begin{array}{c}\beta-\mathrm{NSE}^{\mathrm{c}} \\
\text { median }\end{array}$} & \multirow{2}{*}{$\begin{array}{l}\mathrm{FHV}^{\mathrm{d}} \\
\text { median }\end{array}$} & \multirow{2}{*}{$\begin{array}{c}\text { FMS }^{\mathrm{e}} \\
\text { median }\end{array}$} & \multirow{2}{*}{$\begin{array}{r}F_{L V}{ }^{f} \\
\text { median }\end{array}$} \\
\hline & mean & median & & & & & & \\
\hline EA-LSTM single & $\begin{array}{r}0.674 \\
( \pm 0.006)\end{array}$ & $\begin{array}{r}0.714 \\
( \pm 0.004)\end{array}$ & $\begin{array}{r}2 \\
( \pm 1)\end{array}$ & $\begin{array}{r}0.82 \\
( \pm 0.013)\end{array}$ & $\begin{array}{r}-0.03 \\
( \pm 0.009)\end{array}$ & $\begin{array}{l}-16.9 \\
( \pm 1.1)\end{array}$ & $\begin{array}{l}-10.0 \\
( \pm 1.7)\end{array}$ & $\begin{array}{r}2.0 \\
( \pm 7.6)\end{array}$ \\
\hline EA-LSTM ensemble & 0.705 & 0.742 & 1 & 0.81 & -0.03 & -18.1 & -11.3 & 31.9 \\
\hline LSTM single & $\begin{array}{r}0.685 \\
( \pm 0.015)\end{array}$ & $\begin{array}{r}0.731 \\
( \pm 0.002)\end{array}$ & $\begin{array}{r}1 \\
( \pm 0)\end{array}$ & $\begin{array}{r}0.85 \\
( \pm 0.011)\end{array}$ & $\begin{array}{r}-0.03 \\
( \pm 0.007)\end{array}$ & $\begin{array}{l}-14.8 \\
( \pm 1.1)\end{array}$ & $\begin{array}{r}-8.3 \\
( \pm 1.2)\end{array}$ & $\begin{array}{r}26.5 \\
( \pm 7.6)\end{array}$ \\
\hline LSTM ensemble & 0.718 & 0.758 & 1 & 0.84 & -0.03 & -15.7 & -8.8 & 55.1 \\
\hline SAC-SMA & 0.564 & 0.603 & 13 & 0.78 & -0.07 & -20.4 & -14.3 & 37.3 \\
\hline VIC (basin) & 0.518 & 0.551 & 10 & 0.72 & -0.02 & -28.1 & -6.6 & -70.0 \\
\hline VIC (CONUS) & 0.167 & 0.307 & 41 & 0.46 & -0.07 & -56.5 & -28.0 & 17.4 \\
\hline mHM (basin) & 0.627 & 0.666 & 7 & 0.81 & -0.04 & -18.6 & -7.2 & 11.4 \\
\hline mHM (CONUS) & 0.442 & 0.527 & 29 & 0.59 & -0.04 & -40.2 & -30.4 & 36.4 \\
\hline HBV (lower) & 0.237 & 0.416 & 35 & 0.58 & -0.02 & -41.9 & -15.9 & 23.9 \\
\hline HBV (upper) & 0.631 & 0.676 & 9 & 0.79 & -0.01 & -18.5 & -24.9 & 18.3 \\
\hline FUSE (900) & 0.587 & 0.639 & 12 & 0.80 & -0.03 & -18.9 & -5.1 & -11.4 \\
\hline FUSE (902) & 0.611 & 0.650 & 10 & 0.80 & -0.05 & -19.4 & 9.6 & -33.2 \\
\hline FUSE (904) & 0.582 & 0.622 & 9 & 0.78 & -0.07 & -21.4 & 15.5 & -66.7 \\
\hline $\begin{array}{l}\text { Nash-Sutcliffe efficiency: }( \\
\alpha \text {-NSE decomposition: }(0, \alpha \\
\beta \text {-NSE decomposition: }(-\infty \\
\text { Top } 2 \% \text { peak flow bias: }(-\infty \\
\text { Bias of FDC midsegment slo } \\
30 \%\end{array}$ & $\begin{array}{l}0,1] \text {; values clo } \\
\text {; values close to } \\
\infty) \text {; values close } \\
\infty) \text {; values clos } \\
(-\infty, \infty) \text {; val }\end{array}$ & $\begin{array}{l}r \text { to one are de } \\
\text { ne are desirab } \\
\text { o zero are des } \\
\text { to zero are de } \\
\text { es close to zer }\end{array}$ & $\begin{array}{l}\text { able. } \\
\text { le. } \\
\text { ble. } \\
\text { re desirable. }\end{array}$ & & & & & \\
\hline
\end{tabular}

performing hydrological models. The $\beta$ decomposition of the NSE measures a scaled difference in simulated vs. observed mean streamflow values, and in this case the HBV benchmark performed better that the EA-LSTM, with an average scaled absolute bias (normalized by the root variance of observations) of -0.01 , whereas the EA-LSTM had an average scaled bias of -0.03 for the individual model as well as for the ensemble $\left(p=3.5 \times 10^{-4}\right)$.

\subsection{Robustness and feature ranking}

In Sect. 3.1, we found that adding static features provided a large boost in performance. We would like to check that the model is not simply "remembering" each basin instead of learning a general relation between static features and catchment-specific hydrologic behavior. To this end, we examined model robustness with respect to noisy perturbations of the catchment attributes. Figure 6 shows the results of this experiment by comparing the model performance when forced (not trained) with perturbed static features in each catchment against model performance using the same static feature values that were used for training. As expected, the model performance degrades with increasing noise in the static inputs. However, the degradation does not happen abruptly, but smoothly with increasing levels of noise, which is an indication that the LSTM is not overfitting on the static catchment attributes. That is, it is not remembering each basin with its set of attributes exactly, but rather learns a smooth mapping between attributes and model output. To reiterate from Sect. 2.6.2, the perturbation noise is always relative to the overall standard deviation of the static features across all catchments, which is always $\sigma=1$ (i.e., all static input features were normalized prior to training). When noise with small standard deviation was added (e.g., $\sigma=0.1$ and $\sigma=0.2$ ) the mean and median NSE were relatively stable. The median NSE decreased from 0.71 without noise to 0.48 with an added noise equal to the total variance 
Benchmarking vs. basin-wise calibrated models

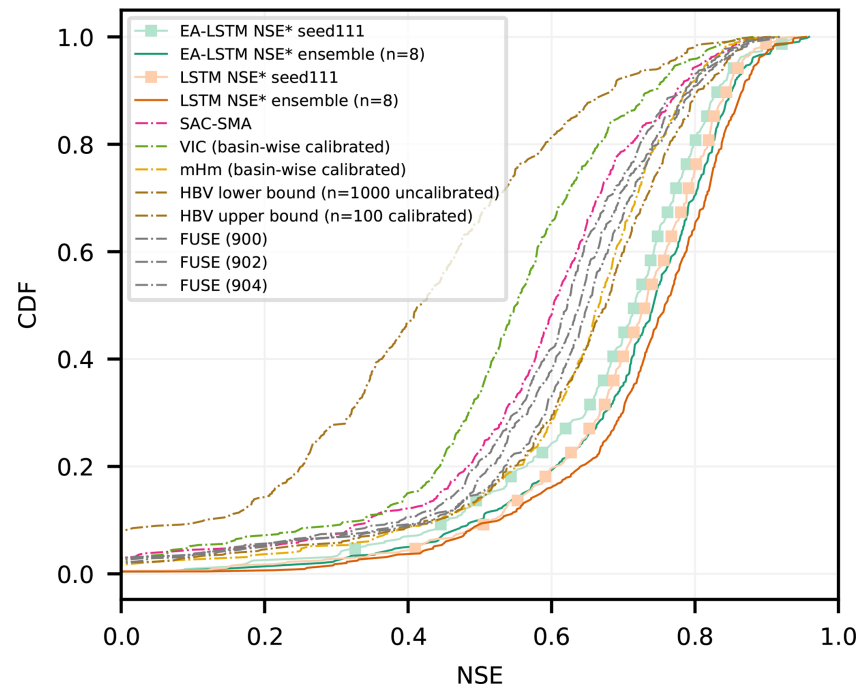

Figure 5. Cumulative density function of the NSE for all basinwise calibrated benchmark models compared to the EA-LSTM and the LSTM with static input features.

of the input features $(\sigma=1)$. This is roughly similar to the performance of the LSTM without static input features (Table 2). In contrast, the lower percentiles of the NSE distributions were more strongly affected by input noise. For example, the 1st (5th) percentile of the NSE values decreased from an NSE of $0.13(0.34)$ to $-5.87(-0.94)$ when going from zero noise (the catchment attribute data from CAMELS) to additive noise with variance equal to the total variance of the inputs (i.e., $\sigma=1$ ). This confirms that static features are especially helpful for increasing performance in basins at the lower end of the NSE spectrum, that is, differentiating hydrological behaviors that are underrepresented in the training dataset.

Figure 7 plots a spatial map where each basin is labeled corresponding to the most sensitive catchment attribute derived from the explicit Morris method for neural networks (Sect. 2.6.2). In the Appalachian Mountains, sensitivity in most catchments is dominated by topological features (e.g., mean catchment elevation and catchment area), and in the eastern US more generally, sensitivity is dominated by climate indices (e.g., mean precipitation, high precipitation duration). Meteorological patterns like aridity and mean precipitation become more important as we move away from the Appalachians and towards the Great Plains, likely because elevation and slope begin to play less of a role. The aridity index dominates sensitivity in the Central Great Plains. In the Rocky Mountains most basins are sensitive climate indices (mean precipitation and high precipitation duration), with some sensitivity to vegetation in the Four Corners region (northern New Mexico). In the West Coast there is a wider variety of dominant sensitivities, reflecting a diversity of catchments.

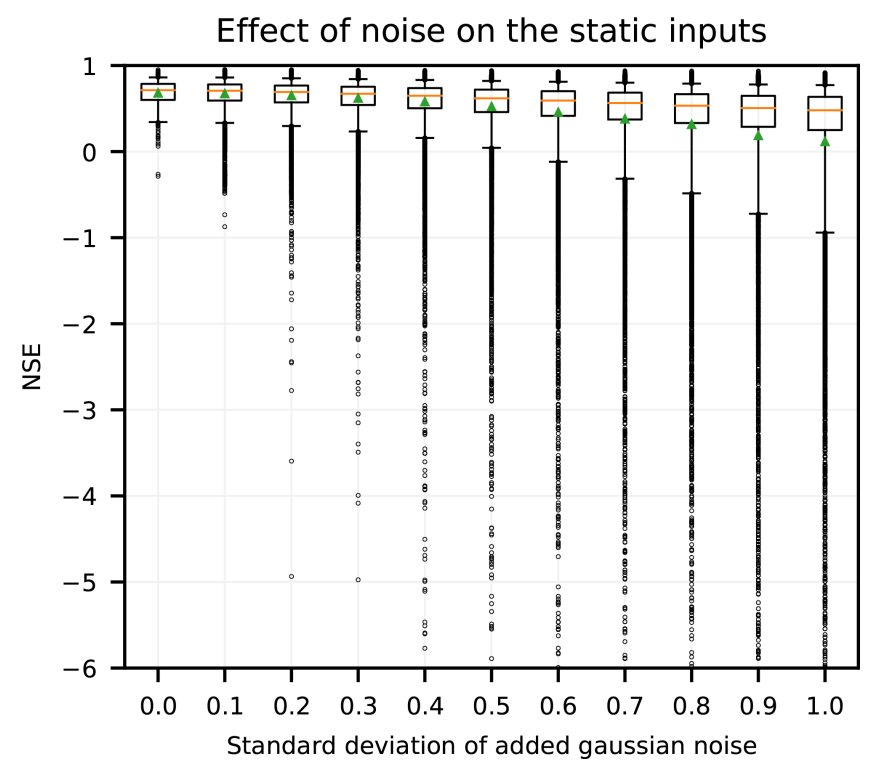

Figure 6. Boxplot showing degradation of model performance with increasing noise level added to the catchment attributes. Orange lines denote the median across catchments, green markers represent means across catchments, box denote the 25 th and 75 th percentiles, whiskers denote the 5 th and 95 th percentiles, and circles are catchments that fall outside the 5th-95th percentile range.

Table 4 provides an overall ranking of dominant sensitivities for one of the eight model repetitions of the EALSTM. These were derived by normalizing the sensitivity measures per basin to the range $(0,1)$ and then calculating the overall mean across all features. As might be inferred from Fig. 7, the most sensitive catchment attributes are topological features (mean elevation and catchment area) and climate indices (mean precipitation, aridity, duration of highprecipitation events, and the fraction of precipitation falling as snow). Certain groups of catchment attributes did not typically provide much additional information. These include vegetation indices like maximum leaf area index or maximum green vegetation fraction as well as the annual vegetation differences. Most soil features were at the lower end of the feature ranking. This sensitivity ranking is interesting in that most of the top-ranked features are relatively easy to measure or estimate globally from readily available gridded data products. Soil maps are one of the hardest features to obtain accurately at a regional scale because they require extensive in situ mapping and interpolation. Note that the results between the eight model repetitions (not shown here) vary slightly in terms of sensitivity values and ranks. However, the quantitative ranking is robust between all eight repetitions, meaning that climate indices (e.g., aridity and mean precipitation) and topological features (e.g., catchment area and mean catchment elevation) are always ranked highest, while soil and vegetation features are of less importance and 


\section{Highest ranked feature per basin}

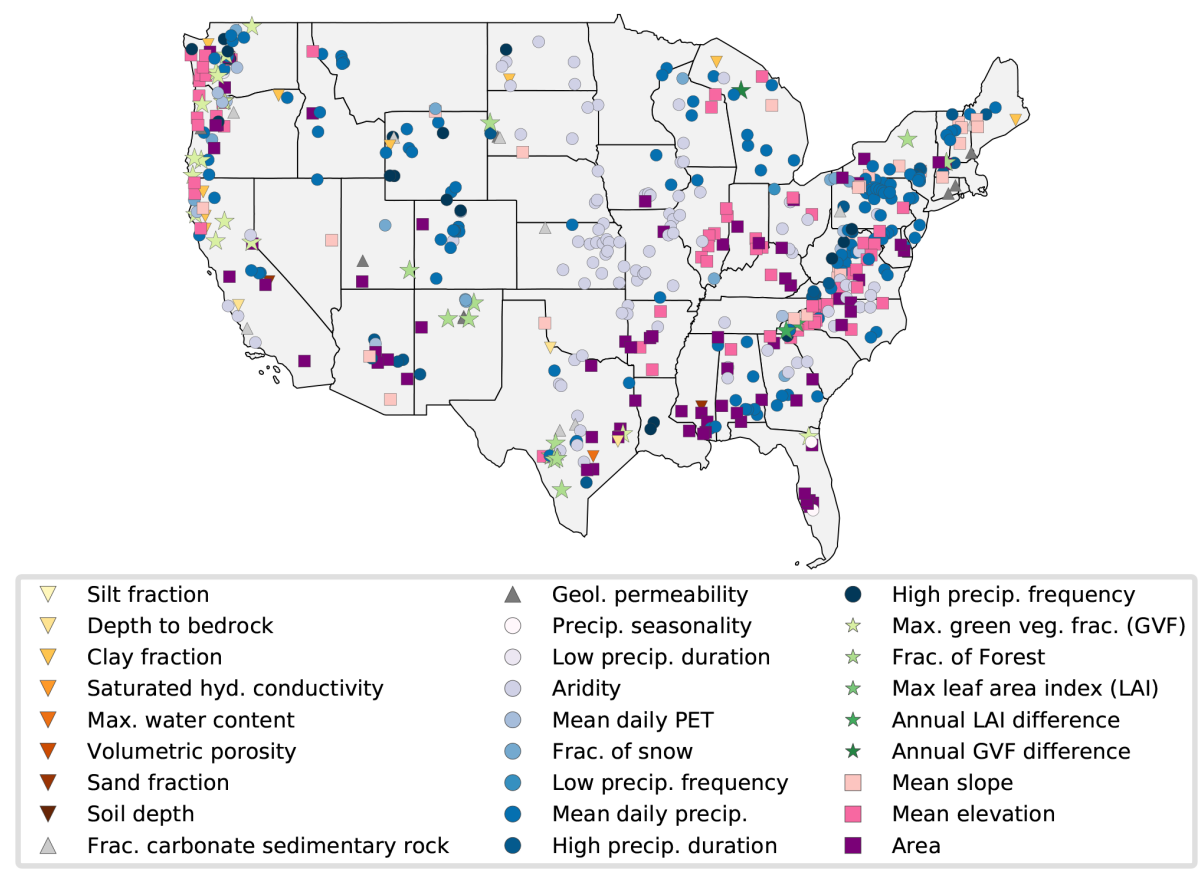

Figure 7. Spatial map of all basins in the dataset. Markers denote the individual catchment characteristic with the highest sensitivity value for each particular basin.

are ranked lower. It is worth noting that our rankings qualitatively agree with much of the analysis by Addor et al. (2018).

\subsection{Analysis of catchment similarity from the embedding layer}

Kratzert et al. (2018a, 2019) showed that these LSTM networks are able to learn to model snow and store this information in specific memory cells without ever directly training on any type of snow-related observation data other than total precipitation and temperature. Multiple types of catchments will use snow-related states in mixture with other states that represent other processes or combinations of processes. The memory cells allow an interpretation along the time axis for each specific basin and are part of both the standard LSTM and the EA-LSTM. A more detailed analysis of the specific functionality of individual cell states is out-of-scope for this paper and will be part of future work. Here, we focus on analysis of the embedding layer, which is a unique feature of the EA-LSTM.

From each of the trained EA-LSTM models, we calculated the input gate vector (Eq. 7) for each basin. The raw EALSTM embedding from one of the models trained over all catchments is shown in Fig. 8. Yellow colors indicate that a particular one of the 256 cell states is activated and contributes to the simulation of a particular catchment. Blue colors indicate that a particular cell state is not used for a partic-

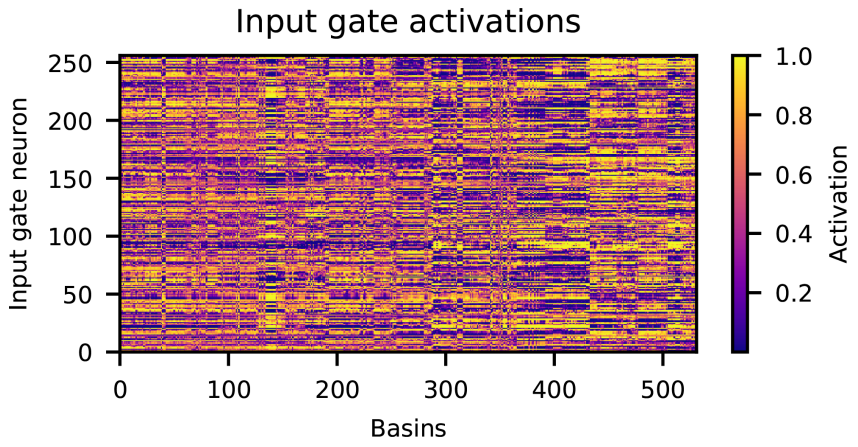

Figure 8. Input gate activations ( $y$ axis) for all 531 basins ( $x$ axis). The basins are ordered from left to right according to the ascending eight-digit USGS gauge ID. Yellow colors denote open input gate cells and blue colors denote closed input gate cells for a particular basin.

ular catchment. These (real-valued) activations are a function of the 27 catchment characteristics input into the static feature layer of the EA-LSTM.

The embedding layer is necessarily high dimensional in this case $\mathbb{R}_{256}$ - due to the fact that the LSTM layer of the model requires sufficient cell states to simulate a wide variety of catchments. Ideally, hydrologically similar catchments should utilize overlapping parts of the LSTM network - this would mean that the network is both learning and us- 
Table 4. Feature ranking derived from the explicit Morris method for one of the EA-LSTM model repetitions.

\begin{tabular}{|c|c|c|}
\hline Rank & Catchment characteristic & Sensitivity \\
\hline 1. & Mean precipitation & 0.68 \\
\hline 2. & Aridity & 0.56 \\
\hline 3. & Area & 0.50 \\
\hline 4. & Mean elevation & 0.46 \\
\hline 5. & High precip. duration & 0.41 \\
\hline 6. & Fraction of snow & 0.41 \\
\hline 7. & High precip. frequency & 0.38 \\
\hline 8. & Mean slope & 0.37 \\
\hline 9. & Geological permeability & 0.35 \\
\hline 10. & Frac. of carbonate sedimentary rock & 0.34 \\
\hline 11. & Clay fraction & 0.33 \\
\hline 12. & Mean PET & 0.31 \\
\hline 13. & Low precip. frequency & 0.30 \\
\hline 14. & Soil depth to bedrock & 0.27 \\
\hline 15. & Precip. seasonality & 0.27 \\
\hline 16. & Frac. of forest & 0.27 \\
\hline 17. & Sand fraction & 0.26 \\
\hline 18. & Saturated hyd. conductivity & 0.24 \\
\hline 19. & Low precip. duration & 0.22 \\
\hline 20. & Max. green veg. frac. (GVF) & 0.21 \\
\hline 21. & Annual GVF diff. & 0.21 \\
\hline 22. & Annual leaf area index (LAI) diff. & 0.21 \\
\hline 23. & Volumetric porosity & 0.19 \\
\hline 24. & Soil depth & 0.19 \\
\hline 25. & Max. LAI & 0.19 \\
\hline 26. & Silt fraction & 0.18 \\
\hline 27. & Max. water content & 0.16 \\
\hline
\end{tabular}

ing catchment similarity to train a regionalizable simulation model.

To assess whether this happened, we first performed a clustering analysis on the $\mathbb{R}_{256}$ embedding space using $k$ means with a Euclidean distance criterion. We compared this with a $k$-means clustering analysis using directly the 27 catchment characteristics to see whether there was a difference in clusters before vs. after the transformation into the embedding layer - remember that this transform was informed by rainfall-runoff training data. To choose an appropriate cluster size, we looked at the mean (and minimum) silhouette value. Silhouette values measure within-cluster similarity and range between $[-1,1]$, with positive values indicating a high degree of separation between clusters and negative values indicating a low degree of separation between clusters. The mean and minimum silhouette values for different cluster sizes are shown in Fig. 9. In all cases with cluster sizes less than 15 , we see that clustering by the values of the embedding layer provides more distinct catchment clusters than when clustering by the raw catchment attributes. This indicates that the EA-LSTM is able to use catchment attribute data to effectively cluster basins into distinct groups.
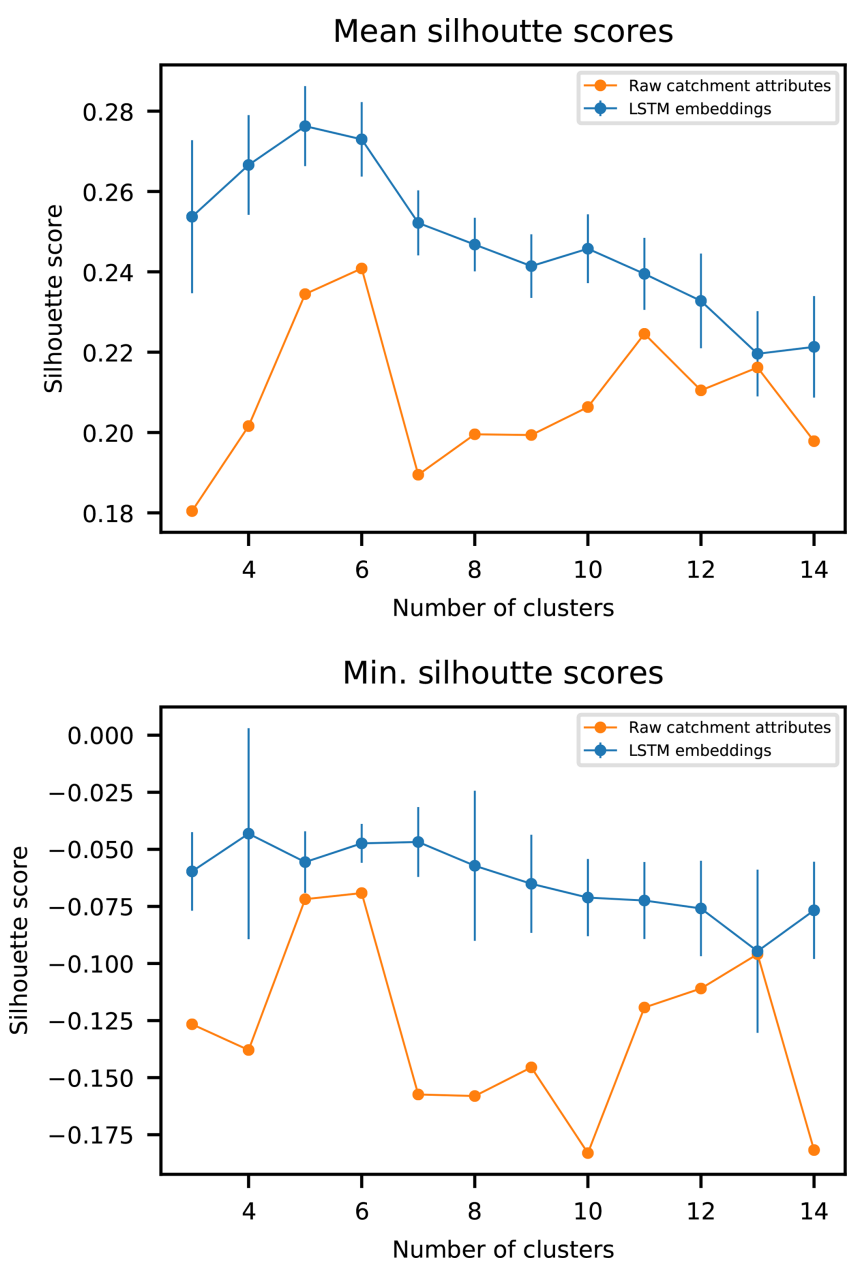

Figure 9. Mean and minimum silhouette scores over varying cluster sizes. For the LSTM embeddings, the line denotes the mean of the $n=8$ repetitions and the vertical lines the standard deviation over 10 random restarts of the $k$-means clustering algorithm.

The highest mean silhouette value from clustering with the raw catchment attributes was $k=6$ and the highest mean silhouette value from clustering with the embedding layer was $k=5$. Ideally, these clusters would be related to hydrologic behavior. To test this, Fig. 10 shows the fractional reduction in variance of 13 hydrologic signatures due to clustering by both raw catchment attributes vs. by the EA-LSTM embedding layer. Ideally, the within-cluster variance of any particular hydrological signature should be as small as possible, so that the fractional reduction in variance is as large (close to one) as possible. In both the $k=5$ and $k=6$ cluster examples, clustering by the EA-LSTM embedding layer reduced variance in the hydrological signatures by more or approximately the same amount as by clustering on the raw catchment attributes. The exception to this was the hfd-mean date, which represents an annual timing process (i.e., the day of year when the catchment releases half of its annual flow). This indicates that the EA-LSTM embedding layer largely 

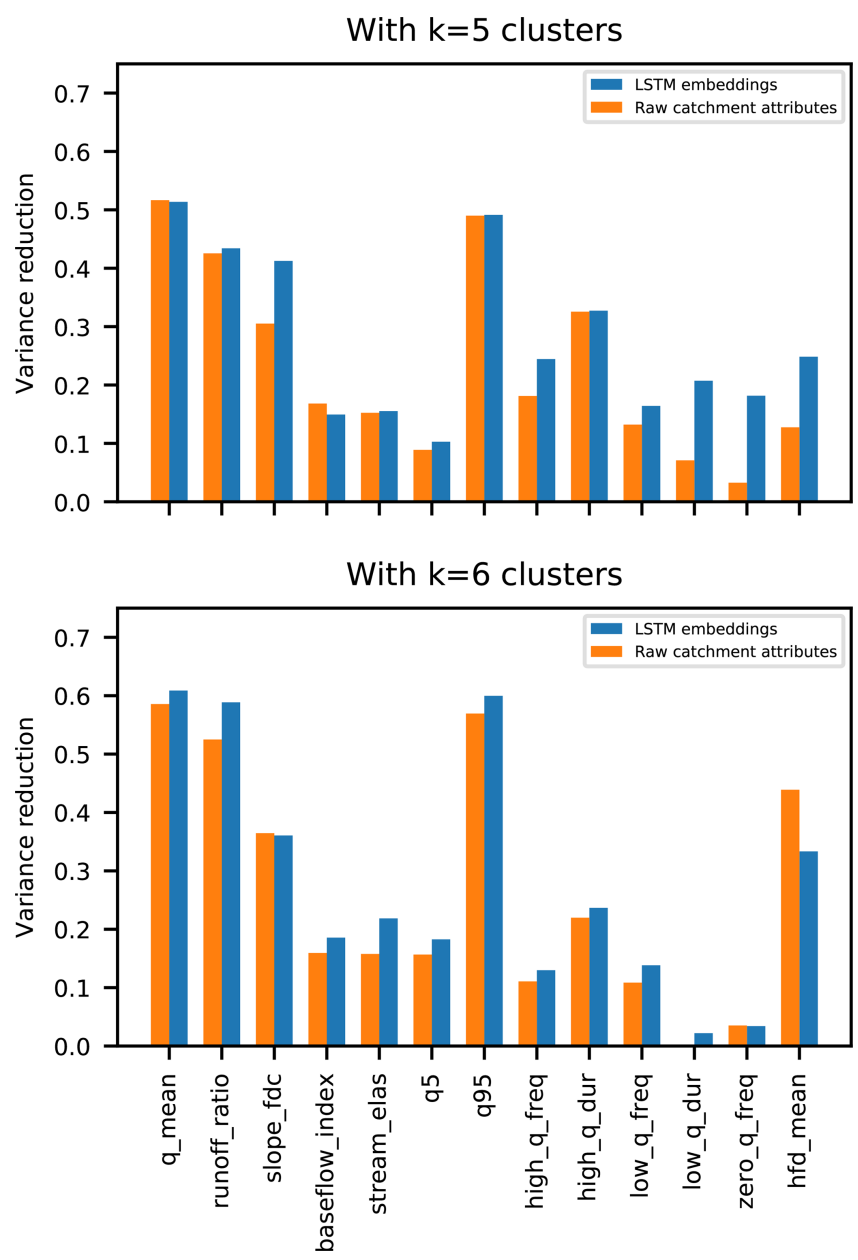

Figure 10. Fractional reduction in variance about different hydrological signatures due to $k$-means clustering on catchment attributes vs. the EA-LSTM embedding layer.

preserves the information content about hydrological behaviors while overall increasing distinctions between groups of similar catchments. The EA-LSTM was able to learn about hydrologic similarity between catchments by directly training on both catchment attributes and rainfall-runoff time series data. Remember that the EA-LSTMs were trained on the time series of streamflow data that these signatures were calculated from, but were not trained directly on these hydrologic signatures.

Clustering maps for $k=5$ and $k=6$ are shown in Fig. 11 . Although latitude and longitude were not part of the catchment attributes vector that was used as input into the embedding layer, both the raw catchment attributes and the embedding layer clearly delineated catchments that correspond to different geographical regions within the CONUS.

To visualize the high-dimensional embedding learned by the EA-LSTM, we used UMAP (McInnes et al., 2018) to project the full $\mathbb{R}_{256}$ embedding onto $\mathbb{R}_{2}$. Figure 12 shows results of the UMAP transformation for one of the eight EA-
LSTMs. In each subplot in Fig. 12, each point corresponds to one basin. The absolute values of the transformed embedding are not of particular interest, but we are interested in the relative arrangement of the basins in this two-dimensional space. Because this is a reduced-dimension transformation, the fact that there are four clear clusters of basins does not necessarily indicate that these are the only distinct basin clusters in our 256-dimensional embedding layer (as we saw above). Figure 12 shows that there is strong interaction between the different catchment characteristics in this embedding layer. For example, high-elevation dry catchments with low forest cover are in the same cluster as low-elevation wet catchments with high forest cover (see cluster B in Fig. 12). These two groups of catchments share parts of their network functionality in the LSTM, whereas highly seasonal catchments activate a different part of the network. Additionally, there are two groups of basins with high forest fractions (clusters A and B); however, if we also consider the mean annual green vegetation difference, both of these clusters are quite distinct. Cluster A in the upper left of each subplot in Fig. 12 contains forest-type basins with a high annual variation in the green vegetation fraction (possibly deciduous forests) and cluster $B$ on the right has almost no annual variation (possibly coniferous forests). One feature that does not appear to affect catchment groupings (i.e., apparently acts independently of other catchment characteristics) is basin size - large and small basins are distributed throughout the three UMAP clusters. To summarize, this analysis demonstrates that the EA-LSTM is able to learn complex interactions between catchment attributes, which allows for grouping of different basins (i.e., choosing which cell states in the LSTM any particular basin or group of basins will use) in ways that account for interaction between different catchment attributes.

\section{Discussion and conclusion}

The EA-LSTM is an example of what Razavi and Coulibaly (2013) called a model-independent method for regional modeling. We cited Besaw et al. (2010) as an earlier example of this type of approach, since they used classical feed-forward neural networks. In our case, the EA-LSTM achieved stateof-the-art results, outperforming multiple locally and regionally calibrated benchmark models. These benchmarking results are arguably a pivotal part of this paper.

The results of the experiments described above demonstrate that a single "universal" deep learning model can learn both regionally consistent and location-specific hydrologic behaviors. The innovation in this study - besides benchmarking the LSTM family of rainfall-runoff models - was to add a static embedding layer in the form of our EA-LSTM. This model offered similar performance as compared with a conventional LSTM (Sect. 3.1) but offers a level of interpretability about how the model learns to differentiate aspects of complex catchment-specific behaviors (Sect. 3.3 and 3.4). In 
(a) LSTM embeddings with $\mathrm{k}=5$

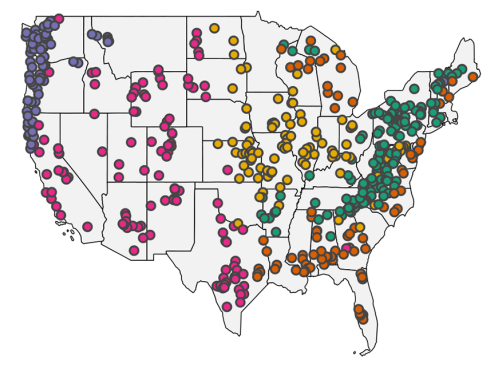

(c)

Raw catchment attributes with $\mathrm{k}=5$

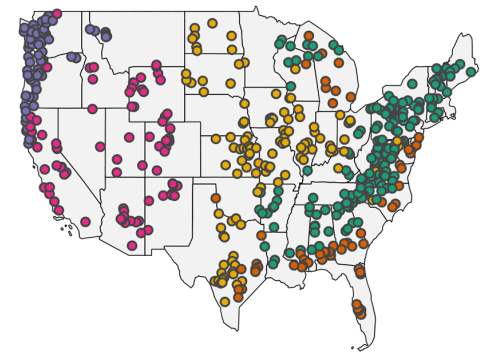

(b) LSTM embeddings with $k=6$

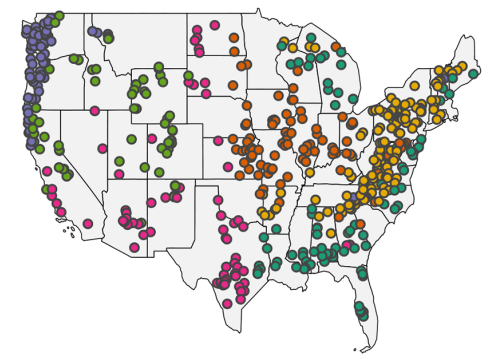

(d)

Raw catchment attributes with $\mathrm{k}=6$

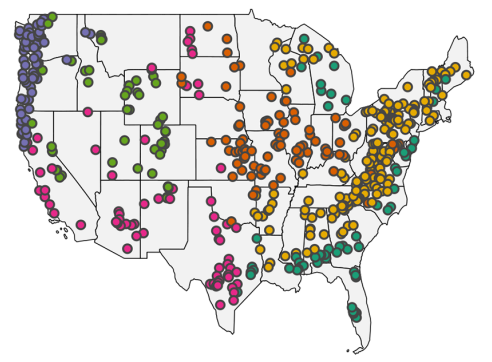

Figure 11. Clustering maps for the LSTM embeddings (a, b) and the raw catchment attributes (c, $\mathbf{d})$ using $k=5$ clusters (a, $\mathbf{c}$, optimal choice for LSTM embeddings) and $k=6$ clusters (b, $\mathbf{d}$, optimal choice for the raw catchment attributes).
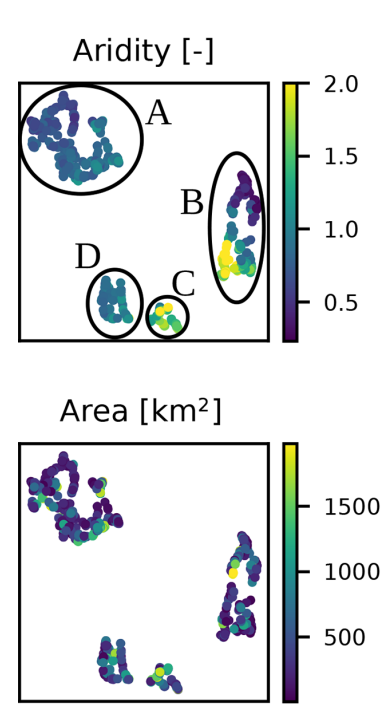

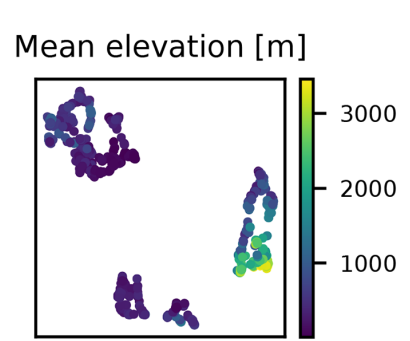

Forest fraction [-]

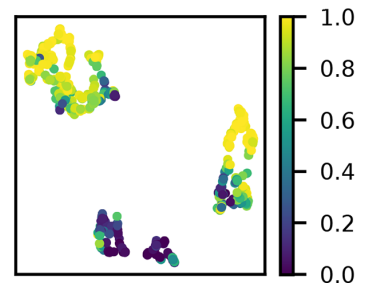

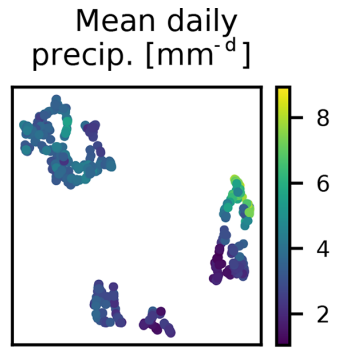

Annual GVF diff. [-]

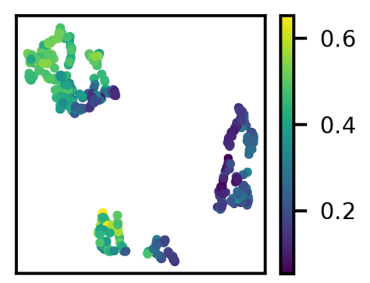

Figure 12. UMAP transformation of the $\mathbb{R}_{256}$ EA-LSTM catchment embedding onto $\mathbb{R}_{2}$. Each dot in each subplot corresponds to one basin. The colors denote specific catchment attributes (notated in subplot titles) for each particular basin. In the upper left plot, clusters are encircled and named to facilitate the description in the text.

a certain sense, this is similar to the aforementioned (MPR) approach, which links its model parameters to the given spatial characteristics (in a nonlinear way, by using transfer functions) but has a fixed model structure to work with. In comparison, our EA-LSTM links catchment characteristics to the dynamics of specific sites and learns the overall model from the combined data of all catchments. Again, the criti- cal take-away, in our opinion, is that the EA-LSTM learns a single model from large catchment datasets in a way that explicitly incorporates local (catchment) similarities and differences.

Neural networks generally require a lot of training data (our unpublished results indicate that it is often difficult to reliably train an LTM) at a single catchment, even with multi- 
decade data records, and adding the ability for the LSTM architecture to transfer information from similar catchments is critical for this to be a viable approach for regional modeling. This is in contrast with traditional hydrological modeling and model calibration, which typically has the best results when models are calibrated independently for each basin. This property of classical models is somewhat problematic, since it has been observed that the spatial patterns of model parameters obtained by ad hoc extrapolations based on calibrated parameters from reference catchments can lead to unrealistic parameter fields and spatial discontinuities of the hydrological states (Mizukami et al., 2017). As shown in Sect. 3.4, this does not occur with our proposed approach. Thus, by leveraging the ability of deep learning to simultaneously learn time series relationships and also spatial relationships in the same predictive framework, we sidestep many problems that are currently associated with the estimation and transfer of hydrologic model parameters.

Moving forward, it is worth mentioning that treating catchment attributes as static is a strong assumption (especially over long time periods), which is not necessarily reflected in the real world. In reality, catchment attributes may continually change at various timescales (e.g., vegetation, topography, pedology, climate). In future studies it will be important to develop strategies to derive analogs to our embedding layer that allow for dynamic or evolving catchment attributes or features - perhaps that act on raw remote sensing data inputs rather than aggregated indexes derived from time series of remote sensing products. In principle, our embedding layer could learn directly from raw brightness temperatures, since there is no requirement that the inputs be hydrologically relevant - only that these inputs are related to hydrological behavior. A dynamic input gate is, at least in principle, possible without significant modification to the proposed EA-LSTM approach, for example, by using a separate sequence-to-sequence LSTM that encodes time-dependent catchment observables (e.g., from climate models or remote sensing) and feeds an embedding layer that is updated at each time step. This would allow the model to "learn" a dynamic embedding that turns off and on different parts of the rainfall-runoff portion of the LSTM over the course of a simulation.
A notable corollary of our main result is that the catchment attributes collected by Addor et al. (2017b) appear to contain sufficient information to distinguish between diverse rainfall-runoff behaviors, at least to a meaningful degree. It is arguable whether this was known previously, since regional modeling studies have largely struggled to fully extract this information (Mizukami et al., 2017) - i.e., existing regional models do not perform with accuracy similarly to models calibrated in a specific catchment. In contrast, our regional EA-LSTM actually performs better than models calibrated separately for individual catchments. This result challenges the idea that runoff time series alone only contain enough information to restrict a handful of parameters (Naef, 1981; Jakeman and Hornberger, 1993; Perrin et al., 2001; Kirchner, 2006) and implies that structural improvements are still possible for most large-scale hydrology models, given the size of today's datasets.

Code and data availability. The CAMELS input data are freely available at the homepage of the NCAR. The validation periods of all benchmark models used in this study are available at https://doi.org/10.4211/hs.474ecc37e7db45baa425cdb4fc1b61e1 (Kratzert, 2019a), the extended Maurer forcings, including daily minimum and maximum temperature, are available at https://doi.org/10.4211/hs.17c896843cf940339c3c3496d0c1c077 (Kratzert, 2019b), our trained networks as well as our model outputs are available at https://doi.org/10.4211/hs.83ea5312635e44dc824eeb99eda12f06 (Kratzert, 2019d), and finally, the code to reproduce our results is available at https://doi.org/10.5281/zenodo.3530884 (Kratzert, 2019c) (https://github.com/kratzert/ealstm_regional_modeling, last access: 13 December 2019). 
Appendix A: Full list of the used CAMELS catchment characteristics

\section{A1 Table of catchment attributes used in this experiment. Description taken from the dataset of Addor et al. (2017a)}

\begin{tabular}{|c|c|}
\hline p_mean & Mean daily precipitation. \\
\hline pet_mean & Mean daily potential evapotranspiration. \\
\hline aridity & Ratio of mean PET to mean precipitation. \\
\hline p_seasonality & $\begin{array}{l}\text { Seasonality and timing of precipitation. Estimated by representing annual } \\
\text { precipitation and temperature as sine waves. Positive (negative) values indicate } \\
\text { precipitation peaks during the summer (winter). Values of approx. } 0 \text { indicate } \\
\text { uniform precipitation throughout the year. }\end{array}$ \\
\hline frac_snow_daily & Fraction of precipitation falling on days with temperatures below $0^{\circ} \mathrm{C}$. \\
\hline high_prec_freq & Frequency of high-precipitation days ( $\geq 5$ times mean daily precipitation). \\
\hline high_prec_dur & $\begin{array}{l}\text { Average duration of high-precipitation events (number of consecutive days with } \\
\geq 5 \text { times mean daily precipitation). }\end{array}$ \\
\hline low_prec_freq & Frequency of dry days $\left(<1 \mathrm{~mm} \mathrm{~d}^{-1}\right)$ \\
\hline low_prec_dur & $\begin{array}{l}\text { Average duration of dry periods (number of consecutive days } \\
\text { with precipitation }<1 \mathrm{~mm} \mathrm{~d}^{-1} \text { ). }\end{array}$ \\
\hline elev_mean & Catchment mean elevation. \\
\hline slope_mean & Catchment mean slope. \\
\hline area_gages2 & Catchment area. \\
\hline forest_frac & Forest fraction. \\
\hline lai_max & Maximum monthly mean of leaf area index. \\
\hline lai_diff & Difference between the max. and min. mean of the leaf area index. \\
\hline gvf_max & Maximum monthly mean of green vegetation fraction. \\
\hline gvf_diff & $\begin{array}{l}\text { Difference between the maximum and minimum monthly } \\
\text { mean of the green vegetation fraction. }\end{array}$ \\
\hline soil_depth_pelletier & Depth to bedrock (maximum $50 \mathrm{~m}$ ). \\
\hline soil_depth_statsgo & Soil depth (maximum $1.5 \mathrm{~m}$ ). \\
\hline soil_porosity & Volumetric porosity. \\
\hline soil_conductivity & Saturated hydraulic conductivity. \\
\hline max_water_content & Maximum water content of the soil. \\
\hline sand_frac & Fraction of sand in the soil. \\
\hline silt_frac & Fraction of silt in the soil. \\
\hline clay_frac & Fraction of clay in the soil. \\
\hline carb_rocks_frac & Fraction of the catchment area characterized as "Carbonate sedimentary rocks". \\
\hline geol_permeability & Surface permeability $(\log 10)$ \\
\hline
\end{tabular}




\section{Appendix B: Hyperparameter tuning}

The hyperparameters, i.e., the number of hidden/cell states, dropout rate, length of the input sequence, and number of stacked LSTM layers for our model, were found by running a grid search over a range of parameter values. Concretely we considered the following possible parameter values.

1. Hidden states: $64,96,128,156,196,224,256$

2. Dropout rate: $0.0,0.25,0.4,0.5$

3. Length of input sequence: $90,180,270,365$

4. Number of stacked LSTM layer: 1,2

We used $k$-fold cross-validation $(k=4)$ to split the basins into a training set and an independent test set. We trained one model for each split for each parameter combination on the combined calibration period of all basins in the specific training set and evaluated the model performance on the calibration data of the test basins. The final configuration was chosen by taking the parameter set that resulted in the highest median NSE over all possible parameter configurations. The parameters are the following.

1. Hidden states: 256

2. Dropout rate: 0.4

3. Length of input sequence length: 270

4. Number of stacked LSTM layer: 1 
Author contributions. FK had the idea for the regional modeling approach. SH proposed the adapted LSTM architecture. FK, DK, and GN designed all the experiments. FK conducted all the experiments and analyzed the results, together with DK, GS, and GN. GN supervised the manuscript from the hydrological perspective and GK and $\mathrm{SH}$ from the machine-learning perspective. GN and $\mathrm{SH}$ share the responsibility for the last authorship in the respective fields. All the authors worked on the manuscript.

Competing interests. The authors declare that they have no conflict of interest.

Acknowledgements. The project relies heavily on open-source software. All programming was done in Python version 3.7 (van Rossum, 1995) and associated libraries, including Numpy (Van Der Walt et al., 2011), Pandas (McKinney, 2010), PyTorch (Paszke et al., 2017), and Matplotlib (Hunter, 2007).

Financial support. This research has been supported by Bosch, ZF, Google, and the NVIDIA Corporation with the GPU donations (LIT (grant no. LIT-2017-3-YOU-003) and FWF (grant no. P 28660N31)).

Review statement. This paper was edited by Nadav Peleg and reviewed by Hoshin Gupta and two anonymous referees.

\section{References}

Addor, N., Newman, A. J., Mizukami, N., and Clark, M. P.: The CAMELS data set: catchment attributes and meteorology for large-sample studies, Hydrol. Earth Syst. Sci., 21, 5293-5313, https://doi.org/10.5194/hess-21-5293-2017, 2017a.

Addor, N., Newman, A. J., Mizukami, N., and Clark, M. P.: Catchment attributes for large-sample studies, UCAR/NCAR, Boulder, CO, USA, https://doi.org/10.5065/D6G73C3Q, 2017b.

Addor, N., Nearing, G., Prieto, C., Newman, A. J., Le Vine, N., and Clark, M. P.: A ranking of hydrological signatures based on their predictability in space, Water Resources Res., 54, 8792-8812, https://doi.org/10.1029/2018WR022606, 2018.

Anderson, E. A.: National Weather Service river forecast system: Snow accumulation and ablation model, NOAA Tech. Memo. NWS HYDRO-17, 87 pp., 1973.

Beck, H. E., van Dijk, A. I. J. M., de Roo, A., Miralles, D. G., McVicar, T. R., Schellekens, J., and Bruijnzeel, L. A.: Global-scale regionalization of hydrologic model parameters, Water Resour. Res., 52, 3599-3622, https://doi.org/10.1002/2015WR018247, 2016.

Besaw, L. E., Rizzo, D. M., Bierman, P. R., and Hackett, W. R.: Advances in ungauged streamflow prediction using artificial neural networks, J. Hydrol., 386, 27-37, 2010.

Beven, K. and Freer, J.: Equifinality, data assimilation, and uncertainty estimation in mechanistic modelling of complex environ- mental systems using the GLUE methodology, J. Hydrol., 249, 11-29, 2001.

Blöschl, G. and Sivapalan, M.: Scale issues in hydrological modelling: a review, Hydrol. Process., 9, 251-290, 1995.

Blöschl, G., Sivapalan, M., Savenije, H., Wagener, T., and Viglione, A.: Runoff prediction in ungauged basins: synthesis across processes, places and scales, Cambridge University Press, Cambridge, 2013.

Burnash, R. J. C.: The NWS river forecast system-catchment modeling, in: Computer models of watershed hydrology, edited by: Singh, V. P., Water Resources Publications, Littleton, CO, 311366, 1995.

Burnash, R. J., Ferral, R. L., and McGuire, R. A.: A generalized streamflow simulation system, conceptual modeling for digital computers, Joint Federal and State River Forecast Center, U.S. National Weather Service, and California Departmentof Water Resources Tech. Rep., 204 pp., 1973.

Clark, M. P., Slater, A. G., Rupp, D. E., Woods, R. A., Vrugt, J. A., Gupta, H. V., Wagener, T., and Hay, L. E.: Framework for Understanding Structural Errors (FUSE): A modular framework to diagnose differences between hydrological models, Water Resour. Res., 44, W00B02, https://doi.org/10.1029/2007WR006735, 2008.

Cohen, J.: Statistical power analysis for the behavioral sciences, 2nd Edn., Erlbaum, Hillsdale, NJ, 1988.

Gupta, H. V., Sorooshian, S., and Yapo, P. O.: Toward improved calibration of hydrologic models: Multiple and noncommensurable measures of information, Water Resour. Res., 34, 751-763, 1998.

Gupta, H. V., Wagener, T., and Liu, Y.: Reconciling theory with observations: elements of a diagnostic approach to model evaluation, Hydrol. Process., 22, 3802-3813, 2008.

Gupta, H. V., Kling, H., Yilmaz, K. K., and Martinez, G. F.: Decomposition of the mean squared error and NSE performance criteria: Implications for improving hydrological modelling, J. Hydrol., 377, 80-91, 2009.

Gupta, H. V., Perrin, C., Blöschl, G., Montanari, A., Kumar, R., Clark, M., and Andréassian, V.: Large-sample hydrology: a need to balance depth with breadth, Hydrol. Earth Syst. Sci., 18, 463477, https://doi.org/10.5194/hess-18-463-2014, 2014.

Henn, B., Clark, M. P., Kavetski, D., and Lundquist, J. D.: Estimating mountain basin-mean precipitation fromstreamflow using Bayesian inference, Water Resour. Res., 51, 8012-8033, 2008.

Herman, J. D., Kollat, J. B., Reed, P. M., and Wagener, T.: Technical Note: Method of Morris effectively reduces the computational demands of global sensitivity analysis for distributed watershed models, Hydrol. Earth Syst. Sci., 17, 2893-2903, https://doi.org/10.5194/hess-17-2893-2013, 2013.

Hochreiter, S.: Untersuchungen zu dynamischen neuronalen Netzen, Diploma, Technische Universität München, Germany, 1991.

Hochreiter, S. and Schmidhuber, J.: Long short-term memory, Neural Comput., 9, 1735-1780, 1997.

Hrachowitz, M., Savenije, H., Blöschl, G., McDonnell, J., Sivapalan, M., Pomeroy, J., Arheimer, B., Blume, T., Clark, M., Ehret, U., Fenicia, F., Freer, J. E., Gelfan, A., Gupta, H. V., Hughes, D. A., Hut, R. W., Montanari, A., Pande, S., Tetzlaff, D., Troch, P. A., Uhlenbrook, S., Wagener, T., Winsemius, H. C., Woods, R. A., Zehe, E., and Cudennec, C.: A decade of Predictions in Ungauged Basins (PUB) - a review, Hydrolog. Sci. J., 58, 1198-1255, 2013. 
Hunter, J. D.: Matplotlib: A 2D graphics environment, Comput. Sci. Eng., 9, 90-95, 2007.

Jakeman, A. J. and Hornberger, G. M.: How much complexity is warranted in a rainfall-runoff model?, Water Resour. Res., 29, 2637-2649, 1993.

Kirchner, J. W.: Getting the right answers for the right reasons: Linking measurements, analyses, and models to advance the science of hydrology, Water Resour. Res., 42, W03S04, https://doi.org/10.1029/2005WR004362, 2006.

Kratzert, F.: Benchmark models, HydroShare, https://doi.org/10. 4211/hs.474ecc37e7db45baa425cdb4fc1b61e1, 2019a.

Kratzert, F.: CAMELS extended Maurer forcings, HydroShare, https://doi.org/10.4211/hs. 17c896843cf940339c3c3496d0c1c077, 2019b.

Kratzert, F.: kratzert/ealstm_regional_modeling: Code to reproduce paper experiments/results, Zenodo, https://doi.org/10.5281/zenodo.3530884, 2019c.

Kratzert, F.: Pre-trained models, HydroShare, https://doi.org/10. 4211/hs.83ea5312635e44dc824eeb99eda12f06, 2019d.

Kratzert, F., Herrnegger, M., Klotz, D., Hochreiter, S., and Klambauer, G.: Do internals of neural networks make sense in the context of hydrology?, in: AGU Fall Meeting Abstracts, 2018AGUFM.H13B..06K, 2018a.

Kratzert, F., Klotz, D., Brenner, C., Schulz, K., and Herrnegger, M.: Rainfall-runoff modelling using Long Short-Term Memory (LSTM) networks, Hydrol. Earth Syst. Sci., 22, 6005-6022, https://doi.org/10.5194/hess-22-6005-2018, 2018 b.

Kratzert, F., Klotz, D., Herrnegger, M., and Hochreiter, S.: A glimpse into the Unobserved: Runoff simulation for ungauged catchments with LSTMs, in: Workshop on Modeling and Decision-Making in the Spatiotemporal Domain, 32nd Conference on Neural Information Processing Systems (NeurIPS 2018), Montreal, Canada, 3-8 December 2018c.

Kratzert, F., Herrnegger, M., Klotz, D., Hochreiter, S., and Klambauer, G.: NeuralHydrology-Interpreting LSTMs in Hydrology, arXiv preprint arXiv:1903.07903, 2019.

Kumar, R., Samaniego, L., and Attinger, S.: Implications of distributed hydrologic model parameterization on water fluxes at multiple scales and locations, Water Resour. Res., 49, 360-379, 2013.

Liang, X., Lettenmaier, D. P., Wood, E. F., and Burges, S. J.: A simple hydrologically based model of land surface water and energy fluxes for general circulation models, J. Geophys. Res., 99, 14415-14428, 1994.

McInnes, L., Healy, J., and Melville, J.: Umap: Uniform manifold approximation and projection for dimension reduction, arXiv preprint arXiv:1802.03426, 2018.

McKinney, W.: Data Structures for Statistical Computing in Python, Proceedings of the 9th Python in Science Conference, Austin, Texas, 28 June-2 July 2010, 1697900, 51-56, 2010.

Mizukami, N., Clark, M. P., Newman, A. J., Wood, A. W., Gutmann, E. D., Nijssen, B., Rakovec, O., and Samaniego, L.: Towards seamless large-domain parameter estimation for hydrologic models, Water Resour. Res., 53, 8020-8040, 2017.

Mizukami, N., Rakovec, O., Newman, A. J., Clark, M. P., Wood, A. W., Gupta, H. V., and Kumar, R.: On the choice of calibration metrics for "high-flow" estimation using hydrologic models, Hydrol. Earth Syst. Sci., 23, 2601-2614, https://doi.org/10.5194/hess-23-2601-2019, 2019.
Morris, M. D.: Factorial sampling plans for preliminary computational experiments, Technometrics, 33, 161-174, 1991.

Naef, F.: Can we model the rainfall-runoff process today?/Peut-on actuellement mettre en modèle le processus pluie-écoulement?, Hydrol. Sci. B., 26, 281-289, 1981.

Nash, J. E. and Sutcliffe, J. V.: River flow forecasting through conceptual models part I - A discussion of principles, J. Hydrol., 10, 282-290, 1970.

Newman, A., Sampson, K., Clark, M., Bock, A., Viger, R., and Blodgett, D.: A large-sample watershed-scale hydrometeorological dataset for the contiguous USA, UCAR/NCAR, Boulder, CO, USA, https://doi.org/10.5065/D6MW2F4D, 2014.

Newman, A. J., Clark, M. P., Sampson, K., Wood, A., Hay, L. E., Bock, A., Viger, R. J., Blodgett, D., Brekke, L., Arnold, J. R., Hopson, T., and Duan, Q.: Development of a large-sample watershed-scale hydrometeorological data set for the contiguous USA: data set characteristics and assessment of regional variability in hydrologic model performance, Hydrol. Earth Syst. Sci., 19, 209-223, https://doi.org/10.5194/hess-19-209-2015, 2015.

Newman, A. J., Mizukami, N., Clark, M. P., Wood, A. W., Nijssen, B., and Nearing, G.: Benchmarking of a physically based hydrologic model, J. Hydrometeorol., 18, 2215-2225, 2017.

Paszke, A., Gross, S., Chintala, S., Chanan, G., Yang, E., DeVito, Z., Lin, Z., Desmaison, A., Antiga, L., and Lerer, A.: Automatic differentiation in PyTorch, in: NIPS 2017 Autodiff Workshop: The Future of Gradient-based Machine Learning Software and Techniques, Long Beach, CA, US, 9 December 2017.

Perrin, C., Michel, C., and Andréassian, V.: Does a large number of parameters enhance model performance? Comparative assessment of common catchment model structures on 429 catchments, J. Hydrol., 242, 275-301, 2001.

Peters-Lidard, C. D., Clark, M., Samaniego, L., Verhoest, N. E. C., van Emmerik, T., Uijlenhoet, R., Achieng, K., Franz, T. E., and Woods, R.: Scaling, similarity, and the fourth paradigm for hydrology, Hydrol. Earth Syst. Sci., 21, 3701-3713, https://doi.org/10.5194/hess-21-3701-2017, 2017.

Prieto, C., Le Vine, N., Kavetski, D., García, E., and Medina, R.: Flow Prediction in Ungauged Catchments Using Probabilistic Random Forests Regionalization and New Statistical Adequacy Tests, Water Resour. Res., 55, 4364-4392, 2019.

Rakovec, O., Mizukami, N., Kumar, R., Newman, A. J., Thober, S., Wood, A. W., Clark, M. P., and Samaniego, L.: Diagnostic Evaluation of Large-domain Hydrologic Models calibrated across the Contiguous United States, J. Geophys. Res.-Atmos., in review, 2019.

Razavi, T. and Coulibaly, P.: Streamflow Prediction in Ungauged Basins: Review of Regionalization Methods, J. Hydrol. Eng., 18, 958-975, 2013.

Saltelli, A., Tarantola, S., Campolongo, F., and Ratto, M.: Sensitivity analysis in practice: a guide to assessing scientific models, Wiley Online Library, 94-100, 2004.

Samaniego, L., Kumar, R., and Attinger, S.: Multiscale parameter regionalization of a grid-based hydrologic model at the mesoscale, Water Resour. Res., 46, W05523, https://doi.org/10.1029/2008WR007327, 2010.

Seibert, J.: Regionalisation of parameters for a conceptual rainfallrunoff model, Agr. Forest Meteorol., 98-99, 279-293, 1999.

Seibert, J. and Vis, M. J. P.: Teaching hydrological modeling with a user-friendly catchment-runoff-model software package, Hydrol. 
Earth Syst. Sci., 16, 3315-3325, https://doi.org/10.5194/hess-163315-2012, 2012.

Seibert, J., Vis, M. J. P., Lewis, E., and van Meerveld, H. J.: Upper and lower benchmarks in hydrological modelling, Hydrol. Process., 32, 1120-1125, 2018.

Sivapalan, M., Takeuchi, K., Franks, S. W., Gupta, V. K., Karambiri, H., Lakshmi, V., Liang, X., McDonnell, J. J., Mendiondo, E. M., O'Connell, P. E., Oki, T., Pomeroy, J. W., Schertzer, D., Uhlenbrook, S., and Zehe, E.: IAHS Decade on Predictions in Ungauged Basins (PUB), 2003-2012: Shaping an exciting future for the hydrological sciences, Hydrolog. Sci. J., 48, 857-880, 2003.

Srivastava, N., Hinton, G., Krizhevsky, A., Sutskever, I., and Salakhutdinov, R.: Dropout: a simple way to prevent neural networks from overfitting, J. Mach. Learn. Res., 15, 1929-1958, 2014.

Van Der Walt, S., Colbert, S. C., and Varoquaux, G.: The NumPy array: A structure for efficient numerical computation, Comput. Sci. Eng., 13, 22-30, 2011. van Rossum, G.: Python tutorial, Technical Report CS-R9526, Centrum voor Wiskunde en Informatica (CWI), Amsterdam, 1995.

Wang, A. and Solomatine, D. P.: Practical Experience of Sensitivity Analysis: Comparing Six Methods, on Three Hydrological Models, with Three Performance Criteria, Water, 11, 1062, https://doi.org/10.3390/w11051062, 2019.

Wilcoxon, F.: Individual comparisons by ranking methods, Biometrics Bull., 1, 80-83, 1945.

Wood, A. W., Maurer, E. P., Kumar, A., and Lettenmaier, D. P.: Long-range experimental hydrologic forecasting for the eastern United States, J. Geophys. Res., 107, 4429, https://doi.org/10.1029/2001JD000659, 2002.

Yilmaz, K. K., Gupta, H. V., and Wagener, T.: A process-based diagnostic approach to model evaluation: Application to the NWS distributed hydrologic model, Water Resour. Res., 44, 1-18, 2008. 